\title{
Finite Element Method for a Kind of Two-Dimensional Space-Fractional Diffusion Equation with Its Implementation
}

\author{
Beiping Duan, Zhoushun Zheng*, Wen Cao \\ School of Mathematics and Statistics, Central South University, Changsha, China \\ Email: zzheng@163.com
}

Received 6 May 2015; accepted 5 June 2015; published 10 June 2015

Copyright (C) 2015 by authors and Scientific Research Publishing Inc.

This work is licensed under the Creative Commons Attribution International License (CC BY). http://creativecommons.org/licenses/by/4.0/

(c) (i) Open Access

\begin{abstract}
In this article, we consider a two-dimensional symmetric space-fractional diffusion equation in which the space fractional derivatives are defined in Riesz potential sense. The well-posed feature is guaranteed by energy inequality. To solve the diffusion equation, a fully discrete form is established by employing Crank-Nicolson technique in time and Galerkin finite element method in space. The stability and convergence are proved and the stiffness matrix is given analytically. Three numerical examples are given to confirm our theoretical analysis in which we find that even with the same initial condition, the classical and fractional diffusion equations perform differently but tend to be uniform diffusion at last.
\end{abstract}

\section{Keywords}

Galerkin Finite Element Method, Symmetric Space-Fractional Diffusion Equation, Stability, Convergence, Implementation

\section{Introduction}

Fractional convection-diffusion equations are generalizations of classical convection-diffusion equations, which have come to be applied in Physics [1]-[4], hydrology [5] [6] and many other fields. As it is difficult to get the analytic solutions of these equations, numerical approaches to different type of fractional convection-diffusion equations are proposed in recent years. Tadjeran et al. [7] considered one-dimensional space-fractional diffusion equation with variable coefficient by fractional Crank-Nicholson method based on the shifted Grünwald formula, and obtained an unconditional stable second-order accurate numerical approximation by extrapolation. Later,

\footnotetext{
${ }^{*}$ Corresponding author.
}

How to cite this paper: Duan, B.P., Zheng, Z.S. and Cao, W. (2015) Finite Element Method for a Kind of Two-Dimensional Space-Fractional Diffusion Equation with Its Implementation. American Journal of Computational Mathematics, 5, 135-157. http://dx.doi.org/10.4236/ajcm.2015.52012 
Tadjeran and Meerschaert [8] utilized the classical alternating directions implicit (ADI) approach with a CrankNicholson discretization and a Richardson extrapolation to solve two-dimensional space-fractional diffusion equation, and proved it is unconditional stable second-order accurate. Sousa [9] derived an implicit second-order accurate numerical method which used a spline approximation for space-fractional diffusion equation and the consistency and stability were examined. A space-time spectral method for time fractional diffusion equation was developed by $\mathrm{Li}$ and $\mathrm{Xu}$ [10], in which the convergence was proven and priori error estimate was given. Xu [11] proposed a discontinuous Galerkin method for one-dimensional convection-subdiffusion equations with fractional Laplace operator and derived stability analysis and optimal convergence rate. Jin et al. [12] gave a full discretization scheme for multi-term time-fractional diffusion equation by using finite difference method in time and finite element method in space, and discussed its stability and error estimate.

The symmetric space-fractional convection-diffusion equation (including both left and right derivatives) was firstly proposed by Chaves [13] to investigate the mechanism of super-diffusion and was later generalized by Benson et al. [14] [15]. It is a powerful approach for a description of transport dynamics in complex systems governed by anomalous diffusion. Zhang [16] et al. considered one-dimensional symmetric space-fractional partial differential equations with Galerkin finite element method in space and a backward difference technique in time, and the stability and convergency were proven. Sousa [17] derived a second order numerical method for one-dimensional symmetric space-fractional convection-diffusion equation and studied its convergence.

Recently, numerical methods for multi-dimensional problems of fractional differential equational are studied. For example, in [18], a semi-alternating direction method for a 2-D fractional reaction diffusion equation are proposed to solve FitzHugh-Nagumo model on an approximate irregular domain. In [19], Crank-Nicolson ADI spectral method is presented to approximate the two-dimensional Riesz space fractional nonlinear reactiondiffusion equation. In [20] [21], Wang and Du proposed fast finite difference methods to compute three-dimensional space-fractional diffusion equations, which reduce the computational cost a lot.

In this paper, we consider the following two-dimensional symmetric space-fractional diffusion equation (SSFDE)

$$
\frac{\partial u(x, y, t)}{\partial t}=a\left(\frac{\partial^{\alpha} u(x, y, t)}{\partial|x|^{\alpha}}+\frac{\partial^{\alpha} u(x, y, t)}{\partial|y|^{\alpha}}\right)+f(x, y, t)
$$

where $1<\alpha \leq 2, \quad a>0$ is a constant, $\frac{\partial^{\alpha} u(x, y, t)}{\partial|x|^{\alpha}}$ and $\frac{\partial^{\alpha} u(x, y, t)}{\partial|y|^{\alpha}}$ are Riesz fractional derivatives defined as follows

$$
\begin{gathered}
\frac{\partial^{\alpha} u(x, y, t)}{\partial|x|^{\alpha}}=\left\{\begin{array}{l}
-\frac{1}{2 \cos \left(\frac{\alpha \pi}{2}\right)}\left({ }_{x} D_{L}^{\alpha}+{ }_{x} D_{R}^{\alpha}\right), n-1<\alpha<n \\
\frac{\partial^{n}}{\partial x^{n}} u(x, y, t), \quad \alpha=n,(n \in N)
\end{array}\right. \\
\frac{\partial^{\alpha} u(x, y, t)}{\partial|y|^{\alpha}}= \begin{cases}-\frac{1}{2 \cos \left(\frac{\alpha \pi}{2}\right)}\left({ }_{y} D_{L}^{\alpha}+{ }_{y} D_{R}^{\alpha}\right), n-1<\alpha<n \\
\frac{\partial^{n}}{\partial y^{n}} u(x, y, t), & \alpha=n,(n \in N)\end{cases}
\end{gathered}
$$

Remark: In this paper, the default fractional derivative is Riemann-Liouville derivative.

This article is organized as follows. In Section 2, we introduce some functional spaces. In Section 3 and Section 4, we prove existence and uniqueness of the variational solution. The full discretization of SSFDE is given in Section 5, where we apply Crank-Nicolson technique in time and Galerkin finite element method in space. Moreover, a detailed stability and convergence analysis is carried out. In section 6, we present the implementation of how to get the stiffness matrix. Finally, some numerical examples are given in Section 7 to confirm our theretical analysis and to compare the difference between fractional diffusion and integer order diffusion system. 


\section{Two-Dimensional Fractional Derivative Spaces}

Ervin and Roop [22] had given the definitions of one-dimensional fractional derivative spaces, and later were generalized to $R^{d}$ via fractional directional integral and derivative in [23]. Here we present some definitions and theorems needed in this paper.

Definition 2.1 (Directional Integral [23]). Let $\mu>0, \theta \in[0,2 \pi)$ be given. The $\mu$ th order fractional integral in the direction of $\theta$ is given by

$$
D_{\theta}^{-\mu} u(x, y):=\frac{1}{\Gamma(\mu)} \int_{0}^{\infty} \xi^{\mu-1} u(x-\xi \cos \theta, y-\xi \sin \theta) \mathrm{d} \xi
$$

Definition 2.2 ([23]). Let $n \in N, \theta \in[0,2 \pi)$ be given. The $n$th order derivative in the direction of $\theta$ is given by

$$
D_{\theta}^{n} u(x, y):=\left(\cos \theta \frac{\partial}{\partial x}+\sin \theta \frac{\partial}{\partial y}\right)^{n} u(x, y)=\left([\cos \theta, \sin \theta]^{t} \cdot \nabla\right)^{n} u(x, y) .
$$

Definition 2.3 (Directional Derivative [23]). Let $\mu>0, \theta \in[0,2 \pi)$ be given. Let $n$ be the smallest integer then $\mu>0, n-1 \leq \mu<n$, and define $\sigma=n-\mu$. Then the $\mu$ th order directional derivative in the direction of $\theta$ is defined by

$$
D_{\theta}^{\mu} u(x, y):=D_{\theta}^{n} D_{\theta}^{-\sigma} u(x, y) .
$$

Definition 2.4 ([23]). Let $\mu>0, \theta \in[0,2 \pi)$ be given. Define the semi-norm

$$
|u|_{J_{L, \theta}^{\mu}(\Omega)}:=\left\|D_{\theta}^{\mu} u\right\|_{L^{2}(\Omega)}
$$

and norm

$$
\|u\|_{J_{L, \theta}^{\mu}(\Omega)}:=\left(\|u\|_{L^{2}(\Omega)}^{2}+|u|_{J_{L, \theta}^{\mu}(\Omega)}^{2}\right)^{1 / 2}
$$

and let $J_{L, \theta}^{\mu}(\Omega)$ denote the closure of $C_{0}^{\infty}(\Omega)$ with respect to $\|\cdot\|_{J_{L, \theta}^{\mu}(\Omega)}$

Definition 2.5 ([23]). Let $\mu>0, \mu \neq n-1 / 2, n \in N, \theta \in[0,2 \pi)$ be given as before. Define the semi-norm

$$
|u|_{J_{S, \theta}^{\mu}(\Omega)}:=\left|\left(D_{\theta}^{\mu} u, D_{\theta+\pi}^{\mu} u\right)_{L^{2}(\Omega)}\right|^{1 / 2}
$$

and norm

$$
\|u\|_{J_{S, \theta}^{\mu}(\Omega)}:=\left(\|u\|_{L^{2}(\Omega)}^{2}+|u|_{J_{S, \theta}^{\mu}(\Omega)}^{2}\right)^{1 / 2}
$$

and let $J_{S, \theta}^{\mu}(\Omega)$ denote the closure of $C_{0}^{\infty}(\Omega)$ with respect to $\|\cdot\|_{J_{S, \theta}^{\mu}(\Omega)}$

Theorem 2.1 ([23]). Let $\mu>0, \mu \neq n-1 / 2, \quad n \in N, \theta \in[0,2 \pi)$ be given. Then the spaces $J_{L, \theta}^{\mu}(\Omega)$ and $J_{S, \theta}^{\mu}(\Omega)$ are equal, with equivalent semi-norms and norms.

Theorem 2.2 ([23]). For $u \in C_{0}^{\infty}(\Omega), \Omega \subset \mathfrak{R}^{2}$, we have

$$
\mathcal{F}\left(D_{\theta}^{\mu} u(x, y)\right)=\left(i \omega_{1} \cos \theta+i \omega_{2} \sin \theta\right)^{\mu} \hat{u}\left(\omega_{1}, \omega_{2}\right),
$$

Definition 2.6 ([23]). Let $\Omega \subset \mathfrak{R}^{2}$ and $\mu>0$. Define the semi-norm

$$
|u|_{H^{\mu}(\Omega)}:=\left\||\omega|^{\mu} \hat{u}\right\|_{L^{2}\left(\Re^{2}\right)}
$$

and norm

$$
\|u\|_{H^{\mu}(\Omega)}:=\left(\|u\|_{L^{2}(\Omega)}^{2}+|u|_{H^{\mu}(\Omega)}^{2}\right)^{1 / 2}
$$


where $\hat{u}$ denotes the Fourier tansform of $u$ with variable $\boldsymbol{\omega}=\left[\omega_{1}, \omega_{2}\right]$. Also let $H_{0}^{\mu}(\Omega)$ denote the closure of $C_{0}^{\infty}(\Omega)$ with respect to $\|\cdot\|$.

In the following, a semi-norm is defined by integral $|\cdot|_{J_{L, \theta}^{\mu}}^{2}(\Omega)$ with respect to the probability measure $M(\mathrm{~d} \theta)$. And

$$
\int_{0}^{2 \pi}|\sin \theta|^{2 \mu} M(\mathrm{~d}(\theta-\psi)) \geq C
$$

holds independent of the value of $\psi \in[-\pi / 2, \pi / 2]$.

Remark: The condition holds if $M(\mathrm{~d} \theta)$ is atomic with at least two atoms, $\theta_{i}, \theta_{j}$, such that $\theta_{i} \neq \theta_{j}+\pi$. In this case, (9) reduces to [23]

$$
\int_{0}^{2 \pi}|\sin \theta|^{2 \mu} M(d(\theta-\psi))=\sum_{i=1}^{n} P\left(\theta=\theta_{i}\right)\left|\sin \left(\theta_{i}+\psi\right)\right|^{2 \mu}
$$

which is positive for all such $\psi$ if and only if $\theta_{i} \neq \theta_{j}+\pi$ for some $i$ and $j$.

Definition 2.7 ([23]) For $\mu>0$, define the semi-norm

$$
|u|_{J_{M}^{\mu}(\Omega)}:=\left(\int_{0}^{2 \pi}|u|_{J_{L, \theta}^{\mu}(\Omega)}^{2} M(\mathrm{~d} \theta)\right)^{1 / 2}
$$

and norm

$$
\|u\|_{J_{M}^{\mu}(\Omega)}:=\left(\|u\|_{L^{2}(\Omega)}^{2}+|u|_{J_{M}^{\mu}(\Omega)}^{2}\right)^{1 / 2}
$$

and let $J_{M}^{\mu}(\Omega)$ denote the closure of $C_{0}^{\infty}(\Omega)$ with respect to $\|\cdot\|_{J_{M}^{\mu}(\Omega)}$.

Theorem 2.3 ([23]). Let $M$ satisfy (9). Then the spaces $H^{\mu}(\Omega)$ and $J_{M}^{\mu}(\Omega)$ are equivalent with equivalent semi-norms and norms.

Theorem 2.4 (Fractional Poincarà Friedrichs Inequality [23]). For $u \in J_{M}^{\mu}(\Omega)$, we have

$$
\|u\|_{J_{M}^{\mu}(\Omega)} \leq \gamma|u|_{J_{M}^{\mu}(\Omega)} .
$$

The definitions and theorems above are basic frame of multi-dimensional fractional derivative spaces. In terms of Equation (1), we let $M$ be atomic with atoms $\left\{\theta_{1}=0, \theta_{2}=\pi / 2\right\}$ or $\left\{\theta_{1}=\pi, \theta_{2}=3 \pi / 2\right\}$, then the seminorm and norm of $J_{M}^{\mu}(\Omega)$ can be defined in the following way:

Definition 2.8 Let $\mu>0$, define the semi-norm

$$
|u|_{J_{M}^{\mu}(\Omega)}=\left(\left\|{ }_{x} D_{L}^{\mu} u\right\|_{L^{2}(\Omega)}^{2}+\|\|_{y} D_{L}^{\mu} u \|_{L^{2}(\Omega)}^{2}\right)^{1 / 2}
$$

or

$$
|u|_{J_{M}^{\mu}(\Omega)}=\left(\left\|{ }_{x} D_{R}^{\mu} u\right\|_{L^{2}(\Omega)}^{2}+\left\|{ }_{y} D_{R}^{\mu} u\right\|_{L^{2}(\Omega)}^{2}\right)^{1 / 2}
$$

and norm

$$
\|u\|_{J_{M}^{\mu}(\Omega)}=\left(\|u\|_{L^{2}(\Omega)}^{2}+|u|_{J_{M}^{\mu}(\Omega)}^{2}\right)^{1 / 2}
$$

It is easy to derive that (12) is equivalent to (13) with using theorem 2.1 and Parseval equality.

Lemma 2.1 (The relationship between R-L and Caputo fractional order derivatives [24]). Assume that the derivatives $f^{(k)}(t),(k=1,2, \cdots, n)$ are continuous in the closed interval $[a, t]$ and $n-1 \leq p<n$, then

$$
{ }_{a} D_{t}^{p} f(t)={ }_{a}^{C} D_{t}^{p} f(t)+\sum_{k=0}^{n-1} \frac{(t-a)^{k-p}}{\Gamma(k+1-p)} f^{k}(a),
$$

where ${ }_{a}^{C} D_{t}^{p}$ denotes Caputo fractional order derivative, which is defined as 


$$
{ }_{a}^{C} D_{t}^{p} f(t)=\frac{1}{\Gamma(n-\alpha)} \int_{a}^{t}(t-\tau)^{n-1-\tau} f^{(n)}(\tau) \mathrm{d} \tau .
$$

So when $f^{(k)}(a)=0$ for $k=0,1, \cdots, n-1$, the two kinds of derivates is equivalent, i.e.

$$
{ }_{a} D_{t}^{p} f(t)={ }_{a}^{C} D_{t}^{p} f(t) .
$$

And if $f^{(k)}(b)=0$ for $k=0,1, \cdots, n-1$, there have ${ }_{t} D_{b}^{p} f(t)={ }_{t}^{C} D_{b}^{p} f(t)$.

Lemma 2.2 ([24]). If $f(t) \in H^{p+q}(a, b)$ and $m-1 \leq p<m, n-1 \leq q<n$, then

$$
{ }_{a} D_{t}^{p}\left({ }_{a} D_{t}^{q} f(t)\right)={ }_{a} D_{t}^{p+q} f(t)-\left.\sum_{j=1}^{n-1}\left[{ }_{a} D_{t}^{q-j} f(t)\right]\right|_{t=a} \frac{(t-a)^{-p-j}}{\Gamma(1-p-j)} .
$$

So, if $f^{(k)}(a)=0$ for $k=0,1, \cdots, n-1$, associating with Lemma 2.1 and the definition of Caputo fractional derivative, it is easy to obtain that

$$
{ }_{a} D_{t}^{p}\left({ }_{a} D_{t}^{q} f(t)\right)={ }_{a} D_{t}^{p+q} f(t)-\sum_{j=1}^{n-1}\left[{ }_{a}^{c} D_{t}^{q-j} f(t)\right] \mid \frac{(t-a)^{-p-j}}{\Gamma(1-p-j)}={ }_{a} D_{t}^{p+q} f(t) .
$$

Lemma 2.3 (Adjoint Property). The left and right Riemann-Liouville fractional integral operator are adjoints in the sense $L^{2}$, i.e., for all $p>0$,

$$
\left({ }_{a} D_{x}^{-p} f, g\right)_{L^{2}(a, b)}=\left(f,{ }_{x} D_{b}^{-p} g\right)_{L^{2}(a, b)}, \quad \forall f, g \in L^{2}(a, b) .
$$

Theorem 2.5 Let $m-1 \leq p<m, n-1 \leq q<n$, and if $f^{(k)}(a)=0$ for $k=0,1, \cdots, n-1, g^{(i)}(b)=0$ for $i=0,1, \cdots, m-1$, then

$$
\left({ }_{a} D_{t}^{p+q} f, g\right)_{L^{2}(a, b)}=\left({ }_{a} D_{t}^{q} f,{ }_{t} D_{b}^{p} g\right)_{L^{2}(a, b)} .
$$

Proof. Let $\sigma=m-p$, combining Lemma 2.2 and Lemma 2.3 we have

$$
\begin{aligned}
\left({ }_{a} D_{t}^{p+q} f, g\right)_{L^{2}(a, b)} & =\left({ }_{a} D_{t}^{p}{ }_{a} D_{t}^{q} f, g\right)_{L^{2}(a, b)}=\left(\left(D^{m}{ }_{a} D_{t}^{-\sigma}\right)_{a} D_{t}^{q} f, g\right)_{L^{2}(a, b)} \\
& =\left({ }_{a} D_{t}^{-\sigma}{ }_{a} D_{t}^{q} f,(-D)^{m} g\right)_{L^{2}(a, b)}=\left({ }_{a} D_{t}^{q} f,{ }_{t} D_{b}^{-\sigma}(-D)^{m} g\right)_{L^{2}(a, b)} \\
& =\left({ }_{a} D_{t}^{q} f,{ }_{t}^{C} D_{b}^{p} g\right)_{L^{2}(a, b)}
\end{aligned}
$$

From Lemma 2.1, we know if $g^{(i)}(b)=0$ for $i=0,1, \cdots, m-1$, then ${ }_{t}^{C} D_{b}^{p} g={ }_{t} D_{b}^{p} g$. So we have

$$
\left({ }_{a} D_{t}^{p+q} f, g\right)_{L^{2}(a, b)}=\left({ }_{a} D_{t}^{q} f,{ }_{t} D_{b}^{p} g\right)_{L^{2}(a, b)}
$$

For convenience, we denote

$$
\left(-\Delta^{*}\right)^{\alpha / 2} u=\frac{1}{2 \cos (\alpha \pi / 2)}\left[\left({ }_{x} D_{L}^{\alpha}+{ }_{x} D_{R}^{\alpha}\right)+\left({ }_{y} D_{L}^{\alpha}+{ }_{y} D_{R}^{\alpha}\right)\right] u .
$$

then Equation (1) can be written in the following form

$$
\left\{\begin{array}{l}
\frac{\partial}{\partial t} u(x, y, t)+a\left(-\Delta^{*}\right)^{\alpha / 2} u(x, y, t)=f(x, y, t) \\
\left.u(x, y, t)\right|_{\partial \Omega}=0, \quad u(x, y, 0)=\varphi(x, y)
\end{array}\right.
$$

where $\alpha \in(1,2], a>0, \Omega \in \mathfrak{R}^{2}$ is an open convex subset.

To derive the variational form of (23), we introduce two properties of $\left(-\Delta^{*}\right)^{\alpha / 2}$ firstly.

Property 1 (Fourier Transform of $\left(-\Delta^{*}\right)^{\alpha / 2}$ ) If $v \in H^{\alpha / 2}\left(\mathfrak{R}^{2}\right)$, then the Fourier Transform of $\left(-\Delta^{*}\right)^{\alpha / 2}$ is 


$$
\mathcal{F}\left(\left(-\Delta^{*}\right)^{\alpha / 2} v\right)=\left(\left|\omega_{1}\right|^{\alpha}+\left|\omega_{2}\right|^{\alpha}\right) \hat{v}\left(\omega_{1}, \omega_{2}\right)
$$

where $\hat{v}\left(\omega_{1}, \omega_{2}\right)$ denotes the Fourier Transform of $v$,

$$
\hat{v}\left(\omega_{1}, \omega_{2}\right)=\iint_{\Re^{2}} \mathrm{e}^{-\left(i \omega_{1} x+i \omega_{2} y\right)} v(x, y) \mathrm{d} x \mathrm{~d} y .
$$

Proof. In view of Theorem 2.1, we can derive the Fourier Transform

$$
\begin{aligned}
& \mathcal{F}\left(\left[\left({ }_{x} D_{L}^{\alpha}+{ }_{x} D_{R}^{\alpha}\right)+\left({ }_{y} D_{L}^{\alpha}+{ }_{y} D_{R}^{\alpha}\right)\right] v\right) \\
& =\left(\left(i \omega_{1}\right)^{\alpha}+\left(-i \omega_{1}\right)^{\alpha}+\left(i \omega_{2}\right)^{\alpha}+\left(-i \omega_{2}\right)^{\alpha}\right) \hat{v}\left(\omega_{1}, \omega_{2}\right) \\
& =\left[\mathrm{e}^{\alpha \ln \left|\omega_{1}\right|}\left(\mathrm{e}^{\alpha i \arg \left(i \omega_{1}\right)}+\mathrm{e}^{\alpha i \arg \left(-i \omega_{1}\right)}\right)+\mathrm{e}^{\alpha \ln \left|\omega_{2}\right|}\left(\mathrm{e}^{\alpha i \arg \left(i \omega_{2}\right)}+\mathrm{e}^{\alpha i \arg \left(-i \omega_{2}\right)}\right)\right] \hat{v}\left(\omega_{1}, \omega_{2}\right) \\
& =\left(\left|\omega_{1}\right|^{\alpha}+\left|\omega_{2}\right|^{\alpha}\right)\left(\mathrm{e}^{i \alpha \pi / 2}+\mathrm{e}^{-i \alpha \pi / 2}\right) \hat{v}\left(\omega_{1}, \omega_{2}\right) \\
& =2 \cos (\alpha \pi / 2)\left(\left|\omega_{1}\right|^{\alpha}+\left|\omega_{2}\right|^{\alpha}\right) \hat{v}\left(\omega_{1}, \omega_{2}\right)
\end{aligned}
$$

Therefore, we have

$$
\mathcal{F}\left(\left(-\Delta^{*}\right)^{\alpha / 2} v\right)=\left(\left|\omega_{1}\right|^{\alpha}+\left|\omega_{2}\right|^{\alpha}\right) \hat{v}\left(\omega_{1}, \omega_{2}\right)
$$

Remark: Here, we use $\left(-\Delta^{*}\right)^{\alpha / 2}$ to make difference from the fractional Laplace operator $(-\Delta)^{\alpha / 2}$, which defined as [25] [26] and its Fourier Transform is $|\boldsymbol{\omega}|^{\alpha} \hat{v}\left(\omega_{1}, \omega_{2}\right)$, where $\boldsymbol{\omega}=\left(\omega_{1}, \omega_{2}\right)$.

Property 2 If $\left.u(x, y, t)\right|_{\partial \Omega}=0,\left.v(x, y, t)\right|_{\partial \Omega}=0$, then

$$
\iint_{\Omega}\left(-\Delta^{*}\right)^{\alpha / 2} u v \mathrm{~d} x \mathrm{~d} y=\frac{1}{2 \cos (\alpha \pi / 2)} \iint_{\Omega} \nabla_{L}^{\alpha / 2} u \nabla_{R}^{\alpha / 2} v+\nabla_{R}^{\alpha / 2} u \nabla_{L}^{\alpha / 2} v \mathrm{~d} x \mathrm{~d} y
$$

where

$$
\nabla_{L}^{\alpha / 2}=\left({ }_{x} D_{L}^{\alpha / 2},{ }_{y} D_{L}^{\alpha / 2}\right), \quad \nabla_{R}^{\alpha / 2}=\left({ }_{x} D_{R}^{\alpha / 2},{ }_{y} D_{R}^{\alpha / 2}\right) .
$$

In fact, when $\alpha=2$, the formula is the classical Green formula.

Proof. Using Theorem 2.5 and taking notice that $\alpha / 2 \in(0,1)$ and $\left.u\right|_{\partial \Omega}=0,\left.v\right|_{\partial \Omega}=0$, we have

$$
\begin{aligned}
& \iint_{\Omega}\left(-\Delta^{*}\right)^{\alpha / 2} u v \mathrm{~d} x \mathrm{~d} y \\
& =\frac{1}{2 \cos (\alpha \pi / 2)} \iint_{\Omega}\left[\left({ }_{x} D_{L}^{\alpha}+{ }_{x} D_{R}^{\alpha}\right)+\left({ }_{y} D_{L}^{\alpha}+{ }_{y} D_{R}^{\alpha}\right)\right] u v \mathrm{~d} x \mathrm{~d} y \\
& =\frac{1}{2 \cos (\alpha \pi / 2)} \iint_{\Omega}\left[\left({ }_{x} D_{L}^{\alpha / 2} u_{x} D_{R}^{\alpha / 2} v+{ }_{y} D_{L}^{\alpha / 2} u_{y} D_{R}^{\alpha / 2} v\right)+\left({ }_{x} D_{R}^{\alpha / 2} u_{x} D_{L}^{\alpha / 2} v+{ }_{y} D_{R}^{\alpha / 2} u_{y} D_{L}^{\alpha / 2} v\right)\right] \mathrm{d} x \mathrm{~d} y \\
& =\frac{1}{2 \cos (\alpha \pi / 2)} \iint_{\Omega} \nabla_{L}^{\alpha / 2} u \nabla_{R}^{\alpha / 2} v+\nabla_{R}^{\alpha / 2} u \nabla_{L}^{\alpha / 2} v \mathrm{~d} x \mathrm{~d} y
\end{aligned}
$$

\section{Variational Formulation}

In order to derive the variational form of (23), we assume $u$ is a sufficiently smooth solution of (23), and multiply by arbitrary $v \in H_{0}^{\alpha / 2}(\Omega)$ to obtain

$$
\iint_{\Omega} \frac{\partial}{\partial t} u(t) v+a\left(-\Delta^{*}\right)^{\alpha / 2} u(t) v \mathrm{~d} x \mathrm{~d} y=\iint_{\Omega} f(t) v \mathrm{~d} x \mathrm{~d} y
$$

The weak formulation of the equation is to find the $u \in H_{0}^{\alpha / 2}(\Omega)$ which can make the following equation established 


$$
\frac{\partial}{\partial t}(u(t), v)+\left(a\left(-\Delta^{*}\right)^{\alpha / 2} u(t), v\right)=(f(t), v), \quad \forall v \in H_{0}^{\alpha / 2}(\Omega) .
$$

With using property 2, the above formula could be written as

$$
\frac{\partial}{\partial t}(u(t), v)+\frac{a}{2 \cos (\alpha \pi / 2)} \iint_{\Omega} \nabla_{L}^{\alpha / 2} u \nabla_{R}^{\alpha / 2} v+\nabla_{R}^{\alpha / 2} u \nabla_{L}^{\alpha / 2} v \mathrm{~d} x \mathrm{~d} y=(f(t), v) .
$$

Thus we define the associated bilinear form $B: H_{0}^{\alpha / 2}(\Omega) \times H_{0}^{\alpha / 2}(\Omega) \rightarrow \Re$ as

$$
B(u, v)=\frac{a}{2 \cos (\alpha \pi / 2)} \iint_{\Omega} \nabla_{L}^{\alpha / 2} u \nabla_{R}^{\alpha / 2} v+\nabla_{R}^{\alpha / 2} u \nabla_{L}^{\alpha / 2} v \mathrm{~d} x \mathrm{~d} y
$$

Theorem 3.1 The form $B(\cdot, \cdot)$ defined by (29) is continuous and coercive.

Proof. According to the definition of $B(u, v)$,

$$
\begin{aligned}
& |B(u, v)|=\frac{a}{|2 \cos (\alpha \pi / 2)|}\left|\iint_{\Omega} \nabla_{L}^{\alpha / 2} u \nabla_{R}^{\alpha / 2} v+\nabla_{R}^{\alpha / 2} u \nabla_{L}^{\alpha / 2} v \mathrm{~d} x \mathrm{~d} y\right| \\
& \leq \frac{a}{|2 \cos (\alpha \pi / 2)|} \iint_{\Omega}\left(\left|{ }_{x} D_{L}^{\alpha / 2} u_{x} D_{R}^{\alpha / 2} v\right|+\left|{ }_{y} D_{L}^{\alpha / 2} u_{y} D_{R}^{\alpha / 2} v\right|+\left|{ }_{x} D_{R}^{\alpha / 2} u_{x} D_{L}^{\alpha / 2} v\right|+\left.\right|_{y} D_{R}^{\alpha / 2} u_{y} D_{L}^{\alpha / 2} v \mid\right) \mathrm{d} x \mathrm{~d} y
\end{aligned}
$$

Using Cauchy-Schwarz inequality we can obtain

$$
\begin{aligned}
|B(u, v)| \leq & \frac{a}{|2 \cos (\alpha \pi / 2)|}\left(\left\|D_{x}^{\alpha / 2} u\right\|_{L^{2}(\Omega)}\left\|_{x} D_{R}^{\alpha / 2} v\right\|_{L^{2}(\Omega)}+\left\|_{y} D_{L}^{\alpha / 2} u\right\|_{L^{2}(\Omega)}\left\|_{y} D_{R}^{\alpha / 2} v\right\|_{L^{2}(\Omega)}\right. \\
& \left.+\left\|D_{x}^{\alpha / 2} u\right\|_{L^{2}(\Omega)}\left\|D_{x}^{\alpha / 2} v\right\|_{L^{2}(\Omega)}+\|\|_{y} D_{R}^{\alpha / 2} u\left\|_{L^{2}(\Omega)}\right\|_{y} D_{L}^{\alpha / 2} v \|_{L^{2}(\Omega)}\right) \\
\leq & \frac{a}{|2 \cos (\alpha \pi / 2)|}\left[\left(\left\|_{x} D_{L}^{\alpha / 2} u\right\|_{L^{2}(\Omega)}+\|\|_{y} D_{L}^{\alpha / 2} u \|_{L^{2}(\Omega)}\right)\left(\|\|_{x} D_{R}^{\alpha / 2} v\left\|_{L^{2}(\Omega)}+\right\|\left\|_{y} D_{R}^{\alpha / 2} v\right\|_{L^{2}(\Omega)}\right)\right. \\
& \left.+\left(\left\|D_{x} D_{R}^{\alpha / 2} u\right\|_{L^{2}(\Omega)}+\left\|_{y} D_{R}^{\alpha / 2} u\right\|_{L^{2}(\Omega)}\right)\left(\left\|_{x} D_{L}^{\alpha / 2} v\right\|_{L^{2}(\Omega)}+\left\|_{y} D_{L}^{\alpha / 2} v\right\|_{L^{2}(\Omega)}\right)\right]
\end{aligned}
$$

Associating the definition of the semi-norm of $J_{M}^{\alpha / 2}(\Omega)$ and using Young's inequality it follows

$$
|B(u, v)| \leq \frac{a}{|\cos (\alpha \pi / 2)|}\left(|u|_{J_{M}^{\alpha / 2}(\Omega)} \cdot|v|_{J_{M}^{\alpha / 2}(\Omega)}+|u|_{J_{M}^{\alpha / 2}(\Omega)} \cdot|v|_{J_{M}^{\alpha / 2}(\Omega)}\right)
$$

So we have

$$
|B(u, v)| \leq \frac{2 a}{|\cos (\alpha \pi / 2)|} \cdot|u|_{J_{M}^{\alpha / 2}(\Omega)}|v|_{J_{M}^{\alpha / 2}(\Omega)} \leq \frac{2 a}{|\cos (\alpha \pi / 2)|} \cdot\|u\|_{J_{M}^{\alpha / 2}(\Omega)}\|v\|_{J_{M}^{\alpha / 2}(\Omega)}
$$

Combining the equivalence of $J_{M}^{\alpha / 2}(\Omega)$ and $H^{\alpha / 2}(\Omega)$, we have

$$
|B(u, v)| \leq \frac{2 a \gamma_{1}}{|\cos (\alpha \pi / 2)|}\|u\|_{H_{0}^{\alpha / 2}(\Omega)}\|v\|_{H_{0}^{\alpha / 2}(\Omega)},
$$

i.e., the form $B(\cdot, \cdot)$ is continuous on $H_{0}^{\alpha / 2}(\Omega) \times H_{0}^{\alpha / 2}(\Omega)$. Replacing $v$ with $u$ in (29), we have

$$
\begin{aligned}
B(u, u) & =\frac{a}{2 \cos (\alpha \pi / 2)} \iint_{\Omega}\left({ }_{x} D_{L}^{\alpha / 2} u_{x} D_{R}^{\alpha / 2} u+{ }_{y} D_{L}^{\alpha / 2} u_{y} D_{R}^{\alpha / 2} u+{ }_{x} D_{R}^{\alpha / 2} u_{x} D_{L}^{\alpha / 2} u+{ }_{y} D_{R}^{\alpha / 2} u_{y} D_{L}^{\alpha / 2} u\right) \mathrm{d} x \mathrm{~d} y \\
& =\frac{a}{\cos (\alpha \pi / 2)} \iint_{\Omega}\left({ }_{x} D_{L}^{\alpha / 2} u_{x} D_{R}^{\alpha / 2} u+{ }_{y} D_{L}^{\alpha / 2} u_{y} D_{R}^{\alpha / 2} u\right) \mathrm{d} x \mathrm{~d} y \\
& =\frac{a}{|\cos (\alpha \pi / 2)|}\left(|u|_{J_{S, 0}^{\alpha / 2}(\Omega)}^{2}+|u|_{J_{S, \pi / 2}^{\alpha / 2}(\Omega)}^{2}\right)
\end{aligned}
$$


According to the equivalence of $J_{L, \theta}^{\mu}(\Omega)$ and $J_{S, \theta}^{\mu}(\Omega)$, and combining Theorem 2.4 we can obtain

$$
B(u, u) \geq \frac{a c \gamma^{2}}{|\cos (\alpha \pi / 2)|}\|u\|_{H_{0}^{\alpha / 2}(\Omega)}^{2},
$$

i.e., the form $B(\cdot, \cdot)$ is coercive on $H_{0}^{\alpha / 2}(\Omega) \times H_{0}^{\alpha / 2}(\Omega)$.

Theorem 3.2 (Energy Inequality). If $u \in L^{2}\left(0, T, H_{0}^{\alpha / 2}(\Omega)\right), \frac{\partial u}{\partial t} \in L^{2}\left(0, T, L^{2}(\Omega)\right), u_{0} \in L^{2}(\Omega)$ and $f \in L^{2}\left(Q_{T}\right)$, where $Q_{T}=(0, T) \times \Omega$. Then, we can obtain the energy estimate

$$
\|u(t)\|_{L^{2}(\Omega)}^{2} \leq\|u(0)\|_{L^{2}(\Omega)}^{2}+\frac{|\cos (\alpha \pi / 2)|}{2 a c \gamma^{2}} \int_{0}^{t}\|f(s)\|_{L^{2}(\Omega)}^{2} \mathrm{~d} s .
$$

i.e. the solution of (23) is well posed.

Proof. Multiply the first formula of (23) by $u$ and integrate both sides of the equation in $\Omega$, then we have

$$
\left(\frac{\partial}{\partial t} u(t), u(t)\right)+B(u(t), u(t))=(f(t), u(t))
$$

As the coercivity of the form $B(\cdot, \cdot)$ and Young's inequality, we obtain

$$
\begin{aligned}
& \frac{1}{2} \frac{\mathrm{d}}{\mathrm{d} t}\|u(t)\|_{L^{2}(\Omega)}^{2}+\frac{a c \gamma^{2}}{|\cos (\alpha \pi / 2)|}\|u(t)\|_{H_{0}^{\alpha / 2}(\Omega)}^{2} \\
& \leq \frac{1}{2 \varepsilon}\|f(t)\|_{L^{2}(\Omega)}^{2}+\frac{\varepsilon}{2}\|u(t)\|_{L^{2}(\Omega)}^{2} \leq \frac{1}{2 \varepsilon}\|f(t)\|_{L^{2}(\Omega)}^{2}+\frac{\varepsilon}{2}\|u(t)\|_{H_{0}^{\alpha / 2}(\Omega)}^{2}
\end{aligned}
$$

Take $\varepsilon=\frac{2 a c \gamma^{2}}{|\cos (\alpha \pi / 2)|}$ and integrating over $(0, t), t \in(0, T]$ to the above inequality we get

$$
\|u(t)\|_{L^{2}(\Omega)}^{2} \leq\|u(0)\|_{L^{2}(\Omega)}^{2}+\frac{|\cos (\alpha \pi / 2)|}{2 a c \gamma^{2}} \int_{0}^{t}\|f(s)\|_{L^{2}(\Omega)}^{2} \mathrm{~d} s
$$

Corollary. The solution of variational formulation (28) exists and is unique.

Proof. The existence can be derived directly from Theorem 3.6 with Lax-Milgram theorem and Theorem 3.7 ensure the uniqueness.

\section{Crank-Nicolson-Galerkin Finite Element Fully Discrete System}

Let $S_{h}$ denote a uniform of partition of $\Omega$, with grid parameter $h$, and $G=\left\{\cup K \mid K \in S_{h}\right\} . V_{h}$ denote the continuous functions on $G$. We define the finite dimensional subspace

$$
X_{h}^{k}:=\left\{v \in C(G):\left.v\right|_{K} \in P_{k}(K), \forall K \in S_{h}\right\}
$$

with the piecewise polynomials $P_{k}$ of order $k(k \in N)$ or less then $k$. Taking a uniform mesh for the time variable $t$ and let

$$
t_{n-\frac{1}{2}}=(n-1 / 2) \tau, t_{n}=n \tau, n=0,1, \cdots, N
$$

where $\tau>0$ being the time step, and $T=t_{N}=N \tau$. Then by the Galerkin finite element method and CrankNicolson technique, (23) is transformed into the following problem: find $u_{h}^{n} \in V_{h}=H_{0}^{\alpha / 2}(\Omega) \cap X_{h}^{k}$ such that

$$
\left\{\begin{array}{l}
\frac{1}{\tau}\left(u_{h}^{n}-u_{h}^{n-1}, v_{h}\right)+B\left(\left(u_{h}^{n}+u_{h}^{n-1}\right) / 2, v_{h}\right)=\left(f\left(t_{n-\frac{1}{2}}\right), v_{h}\right), \forall v_{h} \in V_{h} \\
u_{h}^{0}=u_{0, h}
\end{array}\right.
$$


hold for each $n=1,2, \cdots, N$, where $u_{0, h} \in V_{h}$ is a suitable approximation of initial data $u_{0}$.

Theorem 4.1 For $f \in L^{2}\left(Q_{T}\right)$ and $u_{0} \in L^{2}(\Omega)$, the fully discrete scheme (32) has a unique solution $u_{h}^{n} \in V_{h}$.

Proof. Assume that $B^{\prime}(u, v)=(u, v)+\frac{\tau}{2} B(u, v)$. Then the first formula of (32) can be written as

$$
\begin{aligned}
B^{\prime}\left(u_{h}^{n}, v_{h}\right)= & \left(u_{h}^{n-1}, v_{h}\right)-\frac{\tau}{2} B\left(u_{h}^{n-1}, v_{h}\right) \\
& +\tau\left(f\left(t_{n-\frac{1}{2}}\right), v_{h}\right), \forall v_{h} \in V_{h} .
\end{aligned}
$$

In view of Theorem 4.1, we have

$$
\begin{aligned}
\left|B^{\prime}\left(u_{h}^{n}, v_{h}\right)\right| & =\left|\left(u_{h}^{n}, v_{h}\right)+\frac{\tau}{2} B\left(u_{h}^{n}, v_{h}\right)\right| \leq\left\|u_{h}^{n}\right\|_{L^{2}(\Omega)}\left\|v_{h}\right\|_{L^{2}(\Omega)} \\
& +\frac{a \tau}{|\cos (\alpha \pi / 2)|} \cdot|u|_{H_{0}^{\alpha / 2}(\Omega)}|v|_{H_{0}^{\alpha / 2}(\Omega)} \\
& \leq\left(1+\frac{a \tau}{|\cos (\alpha \pi / 2)|}\right)\left\|u_{h}^{n}\right\|_{H_{0}^{\alpha / 2}(\Omega)}\left\|v_{h}\right\|_{H_{0}^{\alpha / 2}(\Omega)}
\end{aligned}
$$

and

$$
\begin{aligned}
B^{\prime}\left(u_{h}^{n}, u_{h}^{n}\right) & =\left(u_{h}^{n}, u_{h}^{n}\right)+\frac{\tau}{2} B\left(u_{h}^{n}, u_{h}^{n}\right)=\left\|u_{h}^{n}\right\|_{L^{2}(\Omega)}^{2}+\left|\frac{a \tau}{2 \cos (\alpha \pi / 2)}\right|\left|u_{h}^{n}\right|_{H_{0}^{\alpha / 2}(\Omega)}^{2} \\
& \geq \min \left(1, \frac{a \tau}{2|\cos (\alpha \pi / 2)|}\right)\left\|u_{h}^{n}\right\|_{H_{0}^{\alpha / 2}(\Omega)}^{2}
\end{aligned}
$$

Therefore, the bilinear $B^{\prime}(\cdot, \cdot)$ form is continuous over $V_{h} \times V_{h}$ and coercive over $V_{h}$. Furthermore,

$$
\begin{aligned}
& \left|\tau\left(f\left(t_{n-1 / 2}\right), v_{h}\right)-\frac{\tau}{2} B\left(u_{h}^{n-1}, v_{h}\right)+\left(u_{h}^{n-1}, v_{h}\right)\right| \\
& \leq \tau\left\|f\left(t_{n-1 / 2}\right)\right\|_{L^{2}(\Omega)} \cdot\left\|v_{h}\right\|_{H_{0}^{\alpha / 2}(\Omega)}+\left(\frac{a \tau}{|\cos (\alpha \pi / 2)|}+1\right)\left\|u_{h}^{n-1}\right\|_{L^{2}(\Omega)} \cdot\left\|v_{h}\right\|_{L^{2}(\Omega)}
\end{aligned}
$$

i.e., the right side of (33) is continuous. According to Lax-Milgram theorem, the fully discrete approximating system (32) has unique solution $u_{h}^{n} \in V_{h}$.

Theorem 4.2 (Energy Inequality). If $f(t) \in L^{2}\left(Q_{T}\right)$ then the fully discrete approximating system (32) is unconditionally stable and $u_{h}^{n}$ satisfies

$$
\left\|u_{h}^{n}\right\|_{L^{2}(\Omega)} \leq\left\|u_{h}^{0}\right\|_{L^{2}(\Omega)}+T \sup _{t \in[0, T]}\|f(t)\|_{L^{2}(\Omega)}
$$

Proof. Taking $v_{h}=u_{h}^{n}+u_{h}^{n-1}$ in (32), noticing the coercivity of the bilinear form $B(\cdot, \cdot)$ and employing Hölder inequality, we have

$$
\begin{aligned}
\left\|u_{h}^{n}\right\|_{L^{2}(\Omega)}^{2}-\left\|u_{h}^{n-1}\right\|_{L^{2}(\Omega)}^{2} & \leq \tau\left\|f\left(t_{n-\frac{1}{2}}\right)\right\|_{L^{2}(\Omega)}\left\|u_{h}^{n}+u_{h}^{n-1}\right\|_{L^{2}(\Omega)} \\
& \leq \tau\left\|f\left(t_{n-\frac{1}{2}}\right)\right\|_{L^{2}(\Omega)}\left(\left\|u_{h}^{n}\right\|_{L^{2}(\Omega)}+\left\|u_{h}^{n-1}\right\|_{L^{2}(\Omega)}\right)
\end{aligned}
$$

Then we can obtain 


$$
\begin{aligned}
\left\|u_{h}^{n}\right\|_{L^{2}(\Omega)} & \leq\left\|u_{h}^{n-1}\right\|_{L^{2}(\Omega)}+\tau\left\|f\left(t_{n-\frac{1}{2}}\right)\right\|_{L^{2}(\Omega)} \\
& \leq\left\|u_{h}^{n-2}\right\|_{L^{2}(\Omega)}+\tau\left(\left\|f\left(t_{n-\frac{1}{2}}\right)\right\|_{L^{2}(\Omega)}+\left\|f\left(t_{n-1-\frac{1}{2}}\right)\right\|_{L^{2}(\Omega)}\right) \\
& \leq \cdots \leq\left\|u_{h}^{0}\right\|_{L^{2}(\Omega)}+\tau\left(\left\|f\left(t_{n-\frac{1}{2}}\right)\right\|_{L^{2}(\Omega)}+\left\|f\left(t_{n-1-\frac{1}{2}}\right)\right\|_{L^{2}(\Omega)}+\cdots+\left\|f\left(t_{\frac{1}{2}}\right)\right\|_{L^{2}(\Omega)}\right) \\
& \leq\left\|u_{h}^{0}\right\|_{L^{2}(\Omega)}+T \sup _{t \in[0, T]}\|f(t)\|_{L^{2}(\Omega)}
\end{aligned}
$$

So the result is valid.

Lemma 4.1 (Approximation Property [27]) Let $u \in H^{r}(\Omega), 0 \leq r \leq k+1,0 \leq s \leq r$, then there exists $a$ constant $C_{\Omega}$ depending only on $\Omega$ such that

$$
\left\|u-P_{\alpha, h}^{k} u\right\|_{H^{s}(\Omega)} \leq C_{\Omega} h^{r-s}\|u\|_{H^{r}(\Omega)}
$$

where $P_{\alpha, h}^{k}: H^{\alpha}(\Omega) \rightarrow X_{h}^{k}$ is a projection operator.

Theorem 4.3 (Convergence). Assume that $\alpha / 2 \leq r \leq k+1, f \in L^{2}\left(Q_{T}\right), u_{0} \in H^{r}(\Omega)$, and $u$ satisfies $u_{t} \in L^{2}\left(0, T, H^{r}(\Omega)\right), \quad u_{t t} \in L^{2}\left(0, T, H^{\alpha}(\Omega)\right), \quad u_{t t t} \in L^{2}\left(0, T, L^{2}(\Omega)\right)$ Then $u_{n}^{h}$ satisfies

$$
\begin{aligned}
\left\|u_{h}^{n}-u\left(t_{n}\right)\right\|_{L^{2}(\Omega)} \leq & \left\|u_{h}^{0}-u_{0}\right\|_{L^{2}(\Omega)}+C h^{r}\left(\left\|u\left(t_{n}\right)\right\|_{H^{r}(\Omega)}+\int_{t_{0}}^{t_{n}}\left\|u_{t}(t)\right\|_{H^{r}(\Omega)}\right) \\
& +C^{\prime} \tau^{2}\left(\int_{t_{0}}^{t_{n}}\left\|u_{t t t}\right\|_{L^{2}(\Omega)} \mathrm{d} t+\int_{t_{0}}^{t_{n}}\left\|\left(-\Delta^{*}\right)^{\alpha / 2} u_{t t}(t)\right\|_{L^{2}(\Omega)} \mathrm{d} t\right)
\end{aligned}
$$

Proof. Let

$$
e^{n}=u_{h}^{n}-u\left(t_{n}\right)=u_{h}^{n}-P_{\alpha, h}^{k} u+P_{\alpha, h}^{k} u-u\left(t_{n}\right)=\varepsilon^{n}+\eta^{n}
$$

where $P_{\alpha, h}^{k}$ is the elliptic projection operator from $H_{0}^{\alpha / 2}(\Omega)$ into $V_{h}$ which is defined as follows for each $v_{h}$ :

$$
P_{\alpha, h}^{k}(v) \in V_{h}, \quad B\left(P_{\alpha, h}^{k}(v), v_{h}\right)=B\left(v, v_{v}\right) .
$$

Define $\bar{\partial}_{t} \varepsilon^{n}=\frac{\varepsilon^{n}-\varepsilon^{n-1}}{\tau}$, then

$$
\begin{aligned}
& \left(\bar{\partial}_{t} \varepsilon^{n}, v_{h}\right)+B\left(\left(\varepsilon^{n}+\varepsilon^{n-1}\right) / 2, v_{h}\right) \\
& =\left(\bar{\partial}_{t}\left(u_{h}^{n}-P_{\alpha, h}^{k} u\left(t_{n}\right)\right), v_{h}\right)+B\left(\left(u_{h}^{n}-P_{\alpha, h}^{k} u\left(t_{n}\right)+u_{h}^{n-1}-P_{\alpha, h}^{k} u\left(t_{n-1}\right)\right) / 2, v_{h}\right) \\
& =\left(\bar{\partial}_{t} u_{h}^{n}, v_{h}\right)+B\left(\left(u_{h}^{n}+u_{h}^{n-1}\right) / 2, v_{h}\right)-\left(\bar{\partial}_{t} P_{\alpha, h}^{k} u\left(t_{n}\right), v_{h}\right)-B\left(\left(P_{\alpha, h}^{k} u\left(t_{n}\right)+P_{\alpha, h}^{k} u\left(t_{n-1}\right)\right) / 2, v_{h}\right)
\end{aligned}
$$

Looking back to the first formula of (32), we can derive

$$
\begin{aligned}
& \left(\bar{\partial}_{t} \varepsilon^{n}, v_{h}\right)+B\left(\left(\varepsilon^{n}+\varepsilon^{n-1}\right) / 2, v_{h}\right) \\
& =\left(f\left(t_{n-\frac{1}{2}}\right), v_{h}\right)-\left(\bar{\partial}_{t} P_{\alpha, h}^{k} u\left(t_{n}\right), v_{h}\right)-B\left(\left(P_{\alpha, h}^{k} u\left(t_{n}\right)+P_{\alpha, h}^{k} u\left(t_{n-1}\right)\right) / 2, v_{h}\right)
\end{aligned}
$$

Noting that

$$
\left(u_{t}\left(t_{n-\frac{1}{2}}\right), v_{h}\right)+B\left(u\left(t_{n-\frac{1}{2}}\right), v_{h}\right)=\left(f\left(t_{n-\frac{1}{2}}\right), v_{h}\right)
$$


holds for $\forall v \in H_{0}^{\alpha / 2}$, and with using (37), we can obtain

$$
\begin{aligned}
& \left(\bar{\partial}_{t} \varepsilon^{n}, v_{h}\right)+B\left(\left(\varepsilon^{n}+\varepsilon^{n-1}\right) / 2, v_{h}\right) \\
& =\left(u_{t}\left(t_{n-\frac{1}{2}}\right), v_{h}\right)-\left(\bar{\partial}_{t} P_{\alpha, h}^{k} u\left(t_{n}\right), v_{h}\right)+B\left(u\left(t_{n-\frac{1}{2}}\right)-\left(u\left(t_{n}\right)+u\left(t_{n-1}\right)\right) / 2, v_{h}\right) \\
& =\left(u_{t}\left(t_{n-\frac{1}{2}}\right)-\bar{\partial}_{t} P_{\alpha, h}^{k} u\left(t_{n}\right)+\bar{\partial}_{t} u\left(t_{n}\right)-\bar{\partial}_{t} u\left(t_{n}\right), v_{h}\right)+B\left(u\left(t_{n-\frac{1}{2}}\right)-\left(u\left(t_{n}\right)+u\left(t_{n-1}\right)\right) / 2, v_{h}\right) \\
& =-\left(\left(P_{\alpha, h}^{k}-I\right) \bar{\partial}_{t} u\left(t_{n}\right)+\left(\bar{\partial}_{t} u\left(t_{n}\right)-u_{t}\left(t_{n-\frac{1}{2}}\right)\right), v_{h}\right)+B\left(u\left(t_{n-\frac{1}{2}}\right)-\left(u\left(t_{n}\right)+u\left(t_{n-1}\right)\right) / 2, v_{h}\right)
\end{aligned}
$$

Taking $v_{h}=\frac{\varepsilon^{n}+\varepsilon^{n-1}}{2}$, noting $B(u, v)=a \iint_{\Omega}\left(-\Delta^{*}\right)^{\alpha / 2} u v \mathrm{~d} x \mathrm{~d} y$ and combining Cauchy-Schwarz inequality, we can obtain

$$
\begin{aligned}
\frac{1}{2 \tau}\left(\left\|\varepsilon^{n}\right\|_{L^{2}(\Omega)}^{2}-\left\|\varepsilon^{n-1}\right\|_{L^{2}(\Omega)}^{2}\right) \leq & \left|\left(P_{\alpha, h}^{k}-I\right) \overline{\partial_{t}} u\left(t_{n}\right)+\left(\overline{\partial_{t}} u\left(t_{n}\right)-u_{t}\left(t_{n-\frac{1}{2}}\right)\right),\left(\varepsilon^{n}+\varepsilon^{n-1}\right) / 2\right| \\
& +\left|B\left(u\left(t_{n-\frac{1}{2}}\right)-\left(u\left(t_{n}\right)+u\left(t_{n-1}\right)\right) / 2,\left(\varepsilon^{n}+\varepsilon^{n-1}\right) / 2\right)\right| \\
\leq & \frac{1}{2}\left(\left\|\left(P_{\alpha, h}^{k}-I\right) \overline{\partial_{t}} u\left(t_{n}\right)\right\|_{L^{2}(\Omega)}+\left\|\left(\bar{\partial}_{t} u\left(t_{n}\right)-u_{t}\left(t_{n-\frac{1}{2}}\right)\right)\right\|_{L^{2}(\Omega)}\right) \cdot\left\|\varepsilon^{n}+\varepsilon^{n-1}\right\|_{L^{2}(\Omega)} \\
& +\frac{a}{2} \|\left(\left(-\Delta^{*}\right)^{\alpha / 2}\left(u\left(t_{n-\frac{1}{2}}\right)-\left(u\left(t_{n}\right)+u\left(t_{n-1}\right)\right) / 2\right)\left\|_{L^{2}(\Omega)} \cdot\right\|\left(\varepsilon^{n}+\varepsilon^{n-1}\right) \|_{L^{2}(\Omega)}\right. \\
\leq & \frac{1}{2}\left(\left\|\left(P_{\alpha, h}^{k}-I\right) \overline{\partial_{t}} u\left(t_{n}\right)\right\|_{L^{2}(\Omega)}+\left\|\left(\bar{\partial}_{t} u\left(t_{n}\right)-u_{t}\left(t_{n-\frac{1}{2}}\right)\right)\right\|_{L^{2}(\Omega)}\right. \\
& \left.+a\left\|\left(-\Delta^{*}\right)^{\alpha / 2}\left(u\left(t_{n-\frac{1}{2}}\right)-\left(u\left(t_{n}\right)+u\left(t_{n-1}\right)\right) / 2\right)\right\| \|_{L^{2}(\Omega)}\right)\left(\left\|\varepsilon^{n}\right\|_{L^{2}(\Omega)}+\left\|\varepsilon^{n-1}\right\|_{L^{2}(\Omega)}\right)
\end{aligned}
$$

So we have

$$
\begin{aligned}
\left\|\varepsilon^{n}\right\|_{L^{2}(\Omega)}-\left\|\varepsilon^{n-1}\right\|_{L^{2}(\Omega)} \leq & \tau\left(\left\|\left(P_{\alpha, h}^{k}-I\right) \bar{\partial}_{t} u\left(t_{n}\right)\right\|_{L^{2}(\Omega)}+\left\|\left(\bar{\partial}_{t} u\left(t_{n}\right)-u_{t}\left(t_{n-\frac{1}{2}}\right)\right)\right\|_{L^{2}(\Omega)}\right. \\
& \left.+a\left\|\left(-\Delta^{*}\right)^{\alpha / 2}\left(u\left(t_{n-\frac{1}{2}}\right)-\left(u\left(t_{n}\right)+u\left(t_{n-1}\right)\right) / 2\right)\right\|_{L^{2}(\Omega)}\right)
\end{aligned}
$$

In the following we will estimate the three parts of the above inequality respectively. The first part $\left\|\left(P_{\alpha, h}^{k}-I\right) \bar{\partial}_{t} u\left(t_{n}\right)\right\|_{L^{2}(\Omega)}$ satisfies

$$
\begin{aligned}
\left\|\left(P_{\alpha, h}^{k}-I\right) \overline{\partial_{t}} u\left(t_{n}\right)\right\|_{L^{2}(\Omega)} & =\left\|P_{\alpha, h}^{k} \overline{\partial_{t}} u\left(t_{n}\right)-\bar{\partial}_{t} u\left(t_{n}\right)\right\|_{L^{2}(\Omega)} \leq C_{1} h^{r}\left\|\bar{\partial}_{t} u\left(t_{n}\right)\right\|_{H^{r}(\Omega)} \\
& \leq \frac{C_{1} h^{r}}{\tau}\left\|\int_{t_{n-1}}^{t_{n}} u_{t}(t) \mathrm{d} s\right\|_{H^{r}(\Omega)} \leq \frac{C_{1} h^{r}}{\tau} \int_{t_{n-1}}^{t_{n}}\left\|u_{t}(t)\right\|_{H^{r}(\Omega)} \mathrm{d} t
\end{aligned}
$$




$$
\begin{aligned}
& \text { The second part }\left\|\bar{\partial}_{t} u\left(t_{n}\right)-u_{t}\left(t_{n-\frac{1}{2}}\right)\right\|_{L^{2}(\Omega)} \text { satisfies } \\
& \left\|\bar{\partial}_{t} u\left(t_{n}\right)-u_{t}\left(t_{n-\frac{1}{2}}\right)\right\|_{L^{2}(\Omega)}=\frac{1}{2 \tau}\left\|\int_{t_{n-1}}^{t}-\frac{1}{2}\left(t-t_{n-\frac{1}{2}}\right)^{2} u_{t t t}(t) \mathrm{d} t+\int_{t n-\frac{1}{2}}^{t_{n}}\left(t-t_{n-\frac{1}{2}}\right)^{2} u_{t t t}(t) \mathrm{d} t\right\|_{L^{2}(\Omega)} \leq \frac{\tau}{8} \int_{t_{n-1}}^{t_{n}}\left\|u_{t t t}\right\|_{L^{2}(\Omega)} \mathrm{d} t \quad \text { The } \\
& \text { third part }\left\|\left(-\Delta^{*}\right)^{\alpha / 2}\left(u\left(t_{n-\frac{1}{2}}\right)-\left(u\left(t_{n}\right)+u\left(t_{n-1}\right)\right) / 2\right)\right\|_{L^{2}(\Omega)} \text { satisfies } \\
& \left\|\left(-\Delta^{*}\right)^{\alpha / 2}\left(u\left(t_{n-\frac{1}{2}}\right)-\left(u\left(t_{n}\right)+u\left(t_{n-1}\right)\right) / 2\right)\right\|_{L^{2}(\Omega)} \\
& =\left\|\left(-\Delta^{*}\right)^{\alpha / 2} \int_{t_{n-1}}^{t_{n}}\left|t-t_{n-\frac{1}{2}}\right| \cdot u_{t t}(t) \mathrm{d} t\right\|_{L^{2}(\Omega)} \leq \frac{\tau}{2} \int_{t_{n-1}}^{t_{n}}\left\|\left(-\Delta^{*}\right)^{\alpha / 2} u_{t t}(t)\right\|_{L^{2}(\Omega)} \mathrm{d} t
\end{aligned}
$$

Hence we can obtain a recursive inequality

$$
\begin{aligned}
\left\|\varepsilon^{n}\right\|_{L^{2}(\Omega)} & -\left\|\varepsilon^{n-1}\right\|_{L^{2}(\Omega)} \leq C_{1} h^{r} \int_{t_{n-1}}^{t_{n}}\left\|u_{t}(t)\right\|_{H^{r}(\Omega)} \mathrm{d} t \\
& +\frac{\tau^{2}}{8} \int_{t_{n-1}}^{t_{n}}\left\|u_{t t t}\right\|_{L^{2}(\Omega)} \mathrm{d} t+\frac{a \tau^{2}}{2} \int_{t_{n-1}}^{t_{n}}\left\|\left(-\Delta^{*}\right)^{\alpha / 2} u_{t t}(t)\right\|_{L^{2}(\Omega)} \mathrm{d} t
\end{aligned}
$$

Summing up from 1 to $n$ then

$$
\begin{aligned}
\left\|\varepsilon^{n}\right\|_{L^{2}(\Omega)} & \leq\left\|\varepsilon^{0}\right\|_{L^{2}(\Omega)}+C_{1} h^{r} \int_{t_{0}}^{t_{n}}\left\|u_{t}(t)\right\|_{H^{r}(\Omega)} \mathrm{d} t \\
& +\frac{\tau^{2}}{8} \int_{t_{0}}^{t_{n}}\left\|u_{t t t}\right\|_{L^{2}(\Omega)} \mathrm{d} t+\frac{a \tau^{2}}{2} \int_{t_{0}}^{t_{n}}\left\|\left(-\Delta^{*}\right)^{\alpha / 2} u_{t t}(t)\right\|_{L^{2}(\Omega)} \mathrm{d} t
\end{aligned}
$$

Take $s=0$ in (35), then we can obtain

$$
\left\|\eta^{n}\right\|_{L^{2}(\Omega)} \leq C_{\Omega} h^{r}\left\|u\left(t_{n}\right)\right\|_{H^{r}(\Omega)}
$$

From (38) and (39), we can derive the following error estimate

$$
\begin{aligned}
\left\|e^{n}\right\|_{L^{2}(\Omega)}= & \left\|u\left(t_{n}\right)-u_{h}^{n}\right\|_{L^{2}(\Omega)} \leq\left\|\eta^{n}\right\|_{L^{2}(\Omega)}+\left\|\varepsilon^{n}\right\|_{L^{2}(\Omega)} \\
\leq & \left\|u_{h}^{0}-u_{0}\right\|_{L^{2}(\Omega)}+C_{\Omega} h^{r}\left\|u\left(t_{n}\right)\right\|_{H^{r}(\Omega)}+C_{1} h^{r} \int_{t_{0}}^{t_{n}}\left\|u_{t}(t)\right\|_{H^{r}(\Omega)} \mathrm{d} t \\
& +\frac{\tau^{2}}{8} \int_{t_{0}}^{t_{n}}\left\|u_{t t}\right\|_{L^{2}(\Omega)} \mathrm{d} t+\frac{a \tau^{2}}{2} \int_{t_{0}}^{t_{n}}\left\|\left(-\Delta^{*}\right)^{\alpha / 2} u_{t t}(t)\right\|_{L^{2}(\Omega)} \mathrm{d} t
\end{aligned}
$$

Finally, the formula (40) leads to (36).

\section{Computational Implementation}

Since the fractional derivative is a non-local operator, the implementation of finite element method for fractional differential equations is very complex. The main problem is how to obtain the stiffness matrix. In [28], Roop investigated the computational aspects of the Galerkin approximating using continuous piecewise polynomial basis functions on a regular triangulation of the domain. In this section we give the computational details, in which the bilinear functions are chosen as the basis functions. The computational domain is $\Omega=[a, b] \times[c, d]$ and the number of computational grid is $N_{1} \times N_{2}$.

First of all, we consider the problem of finding the fractional derivative of each of the basis function ${ }_{a} D_{x}^{\alpha / 2} \psi_{i}$. The support set of the ith basis function $\psi_{i}$ is $K_{1} \cup K_{2} \cup K_{3} \cup K_{4}$ (see Figure 1). It is defined as 


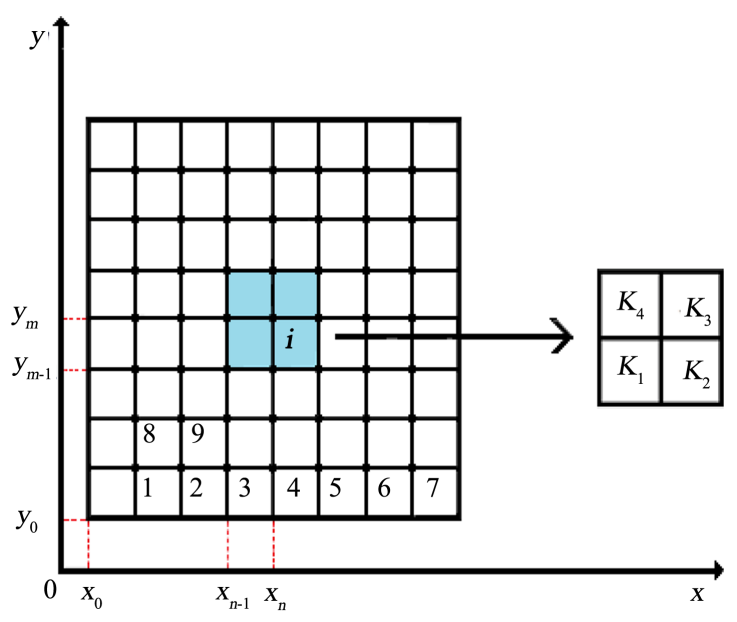

Figure 1. Sketch for the element and node number.

$$
\begin{aligned}
& \left.\psi_{i}\right|_{K_{1}}=\varphi_{1}=\frac{1}{4}\left(1+\frac{x-x_{0}^{1}}{l_{1}}\right)\left(1+\frac{y-y_{0}^{1}}{l_{2}}\right) \\
& \left.\psi_{i}\right|_{K_{2}}=\varphi_{2}=\frac{1}{4}\left(1-\frac{x-x_{0}^{2}}{l_{1}}\right)\left(1+\frac{y-y_{0}^{2}}{l_{2}}\right) \\
& \left.\psi_{i}\right|_{K_{3}}=\varphi_{3}=\frac{1}{4}\left(1-\frac{x-x_{0}^{3}}{l_{1}}\right)\left(1-\frac{y-y_{0}^{3}}{l_{2}}\right) \\
& \left.\psi_{i}\right|_{K_{4}}=\varphi_{4}=\frac{1}{4}\left(1+\frac{x-x_{0}^{4}}{l_{1}}\right)\left(1-\frac{y-y_{0}^{4}}{l_{2}}\right)
\end{aligned}
$$

where $\left(x_{0}^{1}, y_{0}^{1}\right),\left(x_{0}^{2}, y_{0}^{2}\right),\left(x_{0}^{3}, y_{0}^{3}\right),\left(x_{0}^{4}, y_{0}^{4}\right)$ are the centers of the blocks $K_{1}, K_{2}, K_{3}, K_{4}$, and $l_{1}=\frac{x_{n}-x_{n-1}}{2}$, $l_{2}=\frac{y_{m}-y_{m-1}}{2}$. Assume the coordinate of the ith node (see Figure 1) is $\left(x_{n}, y_{m}\right)$, then we can derive

$$
n=i \bmod \left(N_{1}-1\right), \quad m=\frac{i-n}{N_{1}-1}+1 .
$$

If $(x, y) \in K_{1}$, we have

$$
\begin{aligned}
{ }_{a} D_{x}^{\alpha / 2} \psi_{i} & ={ }_{a} D_{x}^{\alpha / 2} \varphi_{1}=\frac{1}{4 l_{1} \Gamma(1-\alpha / 2)} \int_{x_{n-1}}^{x}(x-\xi)^{-\alpha / 2}\left(1+\frac{y-y_{0}^{1}}{l_{2}}\right) \mathrm{d} \xi \\
& =\frac{1}{4 l_{1} \Gamma(2-\alpha / 2)}\left(x-x_{n-1}\right)^{1-\alpha / 2}\left(1+\frac{y-y_{0}^{1}}{l_{2}}\right)
\end{aligned}
$$

If $(x, y) \in K_{2}$, taking notice that $y_{0}^{1}=y_{0}^{2}$, we can get

$$
\begin{aligned}
{ }_{a} D_{x}^{\alpha / 2} \psi_{i}= & { }_{x_{n-1}} D_{x_{n}}^{\alpha / 2} \varphi_{1}+{ }_{x_{n}} D_{x}^{\alpha / 2} \varphi_{2}=\frac{1}{4 l_{1} \Gamma(1-\alpha / 2)} \int_{x_{n-1}}^{x_{n}}(x-\xi)^{-\alpha / 2}\left(1+\frac{y-y_{0}^{1}}{l_{2}}\right) \mathrm{d} \xi \\
& -\frac{1}{4 l_{1} \Gamma(1-\alpha / 2)} \int_{x_{n}}^{x}(x-\xi)^{-\alpha / 2}\left(1+\frac{y-y_{0}^{2}}{l_{2}}\right) \mathrm{d} \xi \\
= & \frac{1}{4 l_{1} \Gamma(2-\alpha / 2)}\left(1+\frac{y-y_{0}^{1}}{l_{2}}\right)\left[\left(x-x_{n-1}\right)^{1-\alpha / 2}-2\left(x-x_{n}\right)^{1-\alpha / 2}\right]
\end{aligned}
$$


If $x>x_{n+1}, y \in\left[y_{m-1}, y_{m}\right]$, we have

$$
\begin{aligned}
{ }_{a} D_{x}^{\alpha / 2} \psi_{i}= & { }_{x_{n-1}} D_{x_{n}}^{\alpha / 2} \varphi_{1}+{ }_{x_{n}} D_{x_{n+1}}^{\alpha / 2} \varphi_{2}=\frac{1}{4 l_{1} \Gamma(1-\alpha / 2)} \int_{x_{n-1}}^{x_{n}}(x-\xi)^{-\alpha / 2}\left(1+\frac{y-y_{0}^{1}}{l_{2}}\right) \mathrm{d} \xi \\
& -\frac{1}{4 l_{1} \Gamma(1-\alpha / 2)} \int_{x_{n}}^{x_{n+1}}(x-\xi)^{-\alpha / 2}\left(1+\frac{y-y_{0}^{2}}{l_{2}}\right) \mathrm{d} \xi \\
= & \frac{1}{4 l_{1} \Gamma(2-\alpha / 2)}\left(1+\frac{y-y_{0}^{1}}{l_{2}}\right)\left[\left(x-x_{n-1}\right)^{1-\alpha / 2}-2\left(x-x_{n}\right)^{1-\alpha / 2}+\left(x-x_{n+1}\right)^{1-\alpha / 2}\right]
\end{aligned}
$$

For $(x, y) \in K_{4}, \quad(x, y) \in K_{3}$ and $x>x_{n+1}, y \in\left[y_{m}, y_{m+1}\right]$, replacing $y_{0}^{1}$ by $y_{0}^{4}$ in the three cases above respectively, we can get ${ }_{a} D_{x}^{\alpha / 2} \psi_{i}$ in the corresponding region.

Secondly, we consider the problem of calculating the inner product $\left({ }_{a} D_{x}^{\alpha / 2} \psi_{i},{ }_{x} D_{b}^{\alpha / 2} \psi_{j}\right)$ for a fixed $i$ and $j=1,2, \cdots, N$, where $N=\left(N_{1}-1\right) \times\left(N_{2}-1\right)$ is the number of inner points. Denote

$$
p=j \bmod \left(N_{1}-1\right), \quad q=\frac{j-n}{N_{1}-1}+1,
$$

then the coordinate of the $j$ th node is $\left(x_{p}, y_{q}\right)$.

It is easy to know when $|m-q| \geq 2$ or $p \leq n-2,\left({ }_{a} D_{x}^{\alpha / 2} \psi_{i},{ }_{x} D_{b}^{\alpha / 2} \psi_{j}\right)=0$. For the other cases, we present the results here. Please see the appendix for the expatiation.

Case 1: $p=n-1, q=m-1$ or $m+1$, i.e. $j=i-\left(N_{1}-1\right)-1$ or $j=i+\left(N_{1}-1\right)-1$

$$
\left({ }_{a} D_{x}^{\alpha / 2} \psi_{i},{ }_{x} D_{b}^{\alpha / 2} \psi_{j}\right)=\frac{l_{2} l_{1}^{1-\alpha}}{12 \Gamma(4-\alpha)} 2^{3-\alpha}
$$

Case 2: $p=n, q=m-1$ or $m+1$, i.e. $j=i-\left(N_{1}-1\right)$ or $j=i+\left(N_{1}-1\right)$

$$
\left({ }_{a} D_{x}^{\alpha / 2} \psi_{i},{ }_{x} D_{b}^{\alpha / 2} \psi_{j}\right)=\frac{l_{2} l_{1}^{1-\alpha}}{12 \Gamma(4-\alpha)}\left(4^{3-\alpha}-4 \cdot 2^{3-\alpha}\right)
$$

Case 3: $p=n+1, q=m-1$ or $m+1$, i.e. $j=i-\left(N_{1}-1\right)+1 \quad$ or $j=i+\left(N_{1}-1\right)+1$

$$
\left({ }_{a} D_{x}^{\alpha / 2} \psi_{i},{ }_{x} D_{b}^{\alpha / 2} \psi_{j}\right)=\frac{l_{2} l_{1}^{1-\alpha}}{12 \Gamma(4-\alpha)}\left(6^{3-\alpha}-4 \cdot 4^{3-\alpha}+6 \cdot 2^{3-\alpha}\right)
$$

Case 4: $p=n+2+k(k=0,1, \cdots), q=m-1$ or $m+1$, i.e. $j=i-\left(N_{1}-1\right)+2+k$ or $j=i+\left(N_{1}-1\right)+2+k$

$$
\left({ }_{a} D_{x}^{\alpha / 2} \psi_{i_{x}} D_{b}^{\alpha / 2} \psi_{j}\right)=\frac{l_{2} l_{1}^{1-\alpha}}{12 \Gamma(4-\alpha)}\left[(8+2 k)^{3-\alpha}-4 \cdot(6+2 k)^{3-\alpha}+6 \cdot(4+2 k)^{3-\alpha}-4 \cdot(2+2 k)^{3-\alpha}+(2 k)^{3-\alpha}\right]
$$

Case 5: $p=n-1, q=m$, i.e. $j=i-1$

$$
\left({ }_{a} D_{x}^{\alpha / 2} \psi_{i},{ }_{x} D_{b}^{\alpha / 2} \psi_{j}\right)=\frac{l_{2} l_{1}^{1-\alpha}}{3 \Gamma(4-\alpha)} 2^{3-\alpha}
$$

Case 6: $p=n, q=m$, i.e. $\quad j=i$

$$
\left({ }_{a} D_{x}^{\alpha / 2} \psi_{i},{ }_{x} D_{b}^{\alpha / 2} \psi_{j}\right)=\frac{l_{2} l_{1}^{1-\alpha}}{3 \Gamma(4-\alpha)}\left(4^{3-\alpha}-4 \cdot 2^{3-\alpha}\right)
$$

Case 7: $p=n+1, q=m$, i.e. $\quad j=i+1$

$$
\left({ }_{a} D_{x}^{\alpha / 2} \psi_{i},{ }_{x} D_{b}^{\alpha / 2} \psi_{j}\right)=\frac{l_{2} l_{1}^{1-\alpha}}{3 \Gamma(4-\alpha)}\left(6^{3-\alpha}-4 \cdot 4^{3-\alpha}+6 \cdot 2^{3-\alpha}\right)
$$

Case 8: $p=n+2+k(k=0,1, \cdots), q=m$, i.e. $\quad j=i+2+k$ 


$$
\left({ }_{a} D_{x}^{\alpha / 2} \psi_{i x} D_{b}^{\alpha / 2} \psi_{j}\right)=\frac{l_{2} 1_{1}^{1-\alpha}}{3 \Gamma(4-\alpha)}\left[(8+2 k)^{3-\alpha}-4 \cdot(6+2 k)^{3-\alpha}+6 \cdot(4+2 k)^{3-\alpha}-4 \cdot(2+2 k)^{3-\alpha}+(2 k)^{3-\alpha}\right]
$$

Finally, we consider the problem of calculating the stiffness matrix $A$ via the inner product obtained. Form Equation (29) we can see that $A$ can be decomposed into four parts $A_{1}, A_{2}, A_{3}, A_{4}$. With ignoring the coefficient $\frac{a}{2 \cos (\alpha \pi / 2)}$ we denote

$$
\begin{array}{ll}
A_{1}(i, j)=\left({ }_{a} D_{x}^{\alpha / 2} \psi_{i},{ }_{x} D_{b}^{\alpha / 2} \psi_{j}\right), & A_{2}(i, j)=\left({ }_{x} D_{b}^{\alpha / 2} \psi_{i},{ }_{a} D_{x}^{\alpha / 2} \psi_{j}\right), \\
A_{3}(i, j)=\left({ }_{c} D_{y}^{\alpha / 2} \psi_{i},{ }_{y} D_{d}^{\alpha / 2} \psi_{j}\right), & A_{4}(i, j)=\left({ }_{y} D_{d}^{\alpha / 2} \psi_{i},{ }_{c} D_{y}^{\alpha / 2} \psi_{j}\right),
\end{array}
$$

then it is obvious that $A_{1}(i, j)=A_{2}(j, i), A_{3}(i, j)=A_{4}(j, i)$, namely $A_{2}=A_{1}^{\mathrm{T}}, A_{4}=A_{3}^{\mathrm{T}}$.

In fact, for $\psi_{i}$ and $\psi_{j}$, if we start numbering these nodes along the direction of $\mathrm{y}$ axis and rename the two basis functions to $\psi_{i_{y}}$ and $\psi_{j_{y}}$, then we have

$$
i_{y}=\left(N_{2}-1\right)(n-1)+m, \quad j_{y}=\left(N_{2}-1\right)(p-1)+q
$$

where $m, n, p, q$ are defined in (41) and (42).

$$
A_{3}(i, j)=\left({ }_{c} D_{y}^{\alpha / 2} \psi_{i},{ }_{y} D_{d}^{\alpha / 2} \psi_{j}\right)=\left({ }_{c} D_{y}^{\alpha / 2} \psi_{i_{y}},{ }_{y} D_{d}^{\alpha / 2} \psi_{j_{y}}\right) \underset{N_{1} \leftrightarrow N_{2}}{\stackrel{l_{1} \leftrightarrow l_{2}}{=}}\left({ }_{a} D_{x}^{\alpha / 2} \psi_{i_{y}},{ }_{x} D_{b}^{\alpha / 2} \psi_{j_{y}}\right)
$$

which means $A_{3}(i, j)$ can be derived from case 1 to case 8 we have presented above with exchanging $l_{1}$ and $l_{2}, \quad N_{1}$ and $N_{2}$. And if $N_{1}=N_{2}, l_{1}=l_{2}$, Equation (44) will reduce to

$$
A_{3}(i, j)=\left({ }_{a} D_{x}^{\alpha / 2} \psi_{i_{y}},{ }_{x} D_{b}^{\alpha / 2} \psi_{j_{y}}\right)=A_{1}\left(i_{y}, j_{y}\right) \text {. }
$$

\section{Numerical Experiments}

Example 1. Consider the following problem:

$$
\left\{\begin{array}{l}
\frac{\partial}{\partial t} u=a\left(\frac{\partial^{\alpha} u}{\partial|x|^{\alpha}}+\frac{\partial^{\alpha} u}{\partial|y|^{\alpha}}\right)+f, \text { in } Q_{T}=(0, T] \times \Omega \\
\left.u(x, y, t)\right|_{\partial \Omega}=0 \\
u(x, y, 0)=500\left(0.25-x^{2}\right)^{2}\left(0.25-y^{2}\right)^{2} \text {, on } \Omega=(-0.5,0.5) \times(-0.5,0.5)
\end{array}\right.
$$

Which has exact solution $u(x, y, t)=500 \mathrm{e}^{-t}\left(1-x^{2}\right)^{2}\left(1-y^{2}\right)^{2}$, and

$$
\begin{aligned}
f(x, y, t)= & -500 \mathrm{e}^{-t}\left(0.25-x^{2}\right)^{2}\left(0.25-y^{2}\right)^{2}+\frac{1000 \mathrm{e}^{-t}\left(0.25-y^{2}\right)^{2}}{\cos (\alpha \pi / 2)} a\left[\frac{(0.5+x)^{2-\alpha}+(0.5-x)^{2-\alpha}}{\Gamma(3-\alpha)}\right. \\
& \left.-6 \frac{(0.5+x)^{3-\alpha}+(0.5-x)^{3-\alpha}}{\Gamma(4-\alpha)}+12 \frac{(0.5+x)^{4-\alpha}+(0.5-x)^{4-\alpha}}{\Gamma(5-\alpha)}\right] \\
& +\frac{1000 \mathrm{e}^{-t}\left(0.25-x^{2}\right)^{2}}{\cos (\alpha \pi / 2)} a\left[\frac{(0.5+y)^{2-\alpha}+(0.5-y)^{2-\alpha}}{\Gamma(3-\alpha)}\right. \\
& \left.-6 \frac{(0.5+y)^{3-\alpha}+(0.5-y)^{3-\alpha}}{\Gamma(4-\alpha)}+12 \frac{(0.5+y)^{4-\alpha}+(0.5-y)^{4-\alpha}}{\Gamma(5-\alpha)}\right]
\end{aligned}
$$

Obviously, $u(t) \in H_{0}^{\alpha / 2}(\Omega) \cap H^{2}(\Omega)$. We take $\alpha=1.6$ and 1.9 respectively, then present corresponding 
experimental error and convergence rate in $L_{2}$ norm in Table 1 with $\tau=0.01, a=5, T=0.5$. To display the numerical solution and error visually, we present the surfaces of $u_{h}^{n}$ and $u_{h}^{n}-u$ at $t=T=0.5$ in Figure 2 and Figure 3 with $h_{1}=h_{2}=1 / 64$.

Remark: The trial function in all of the numerical experiments is bilinear function.

We can see that the results support our error estimate and ensure the numerical approximation is effective. In the following, we take fixed initial value and source term independent of $\alpha$ to try to describe the character of the solution with the change of $\alpha$.

Example 2. Consider the following problem

Table 1. Experimental error and convergence rate in $L_{2}$ norm.

\begin{tabular}{ccccc}
\hline$h_{1}=h_{2}$ & $\left\|u-u_{h}^{N}\right\|_{L_{2}(\Omega)}(\alpha=1.6)$ & Cvge. rate & $\left\|u-u_{h}^{N}\right\|_{L_{2}(\Omega)}(\alpha=1.9)$ & Cvge. rate \\
$1 / 8$ & $1.783209495 \times 10^{-2}$ & & $1.189074481 \times 10^{-2}$ & \\
$1 / 16$ & $4.854890964 \times 10^{-3}$ & 1.88 & $3.268507092 \times 10^{-3}$ & 1.86 \\
$1 / 32$ & $1.270768629 \times 10^{-3}$ & 1.93 & $8.732931724 \times 10^{-3}$ & 1.90 \\
$1 / 64$ & $3.237092091 \times 10^{-4}$ & 1.97 & $2.271818797 \times 10^{-4}$ & 1.94 \\
\hline
\end{tabular}
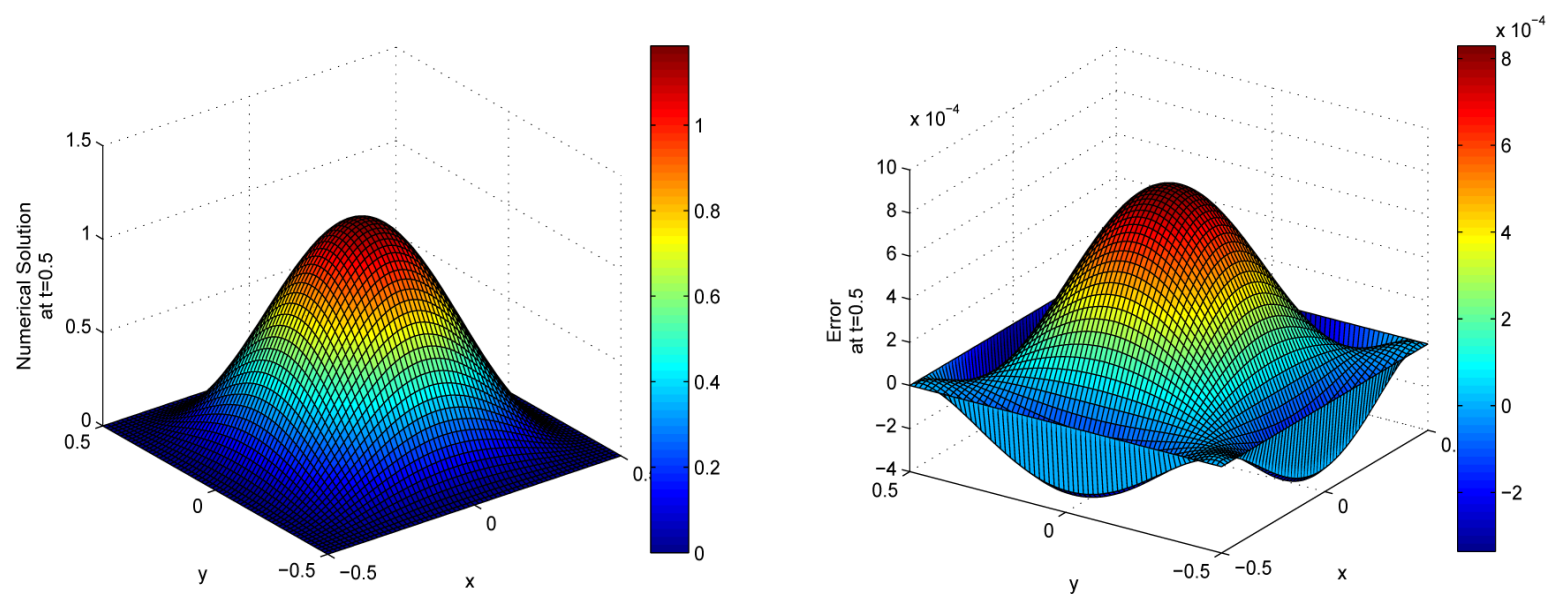

Figure 2. Numerical solution (left) and error (right) for $\alpha=1: 6$ at $t=0.5$.
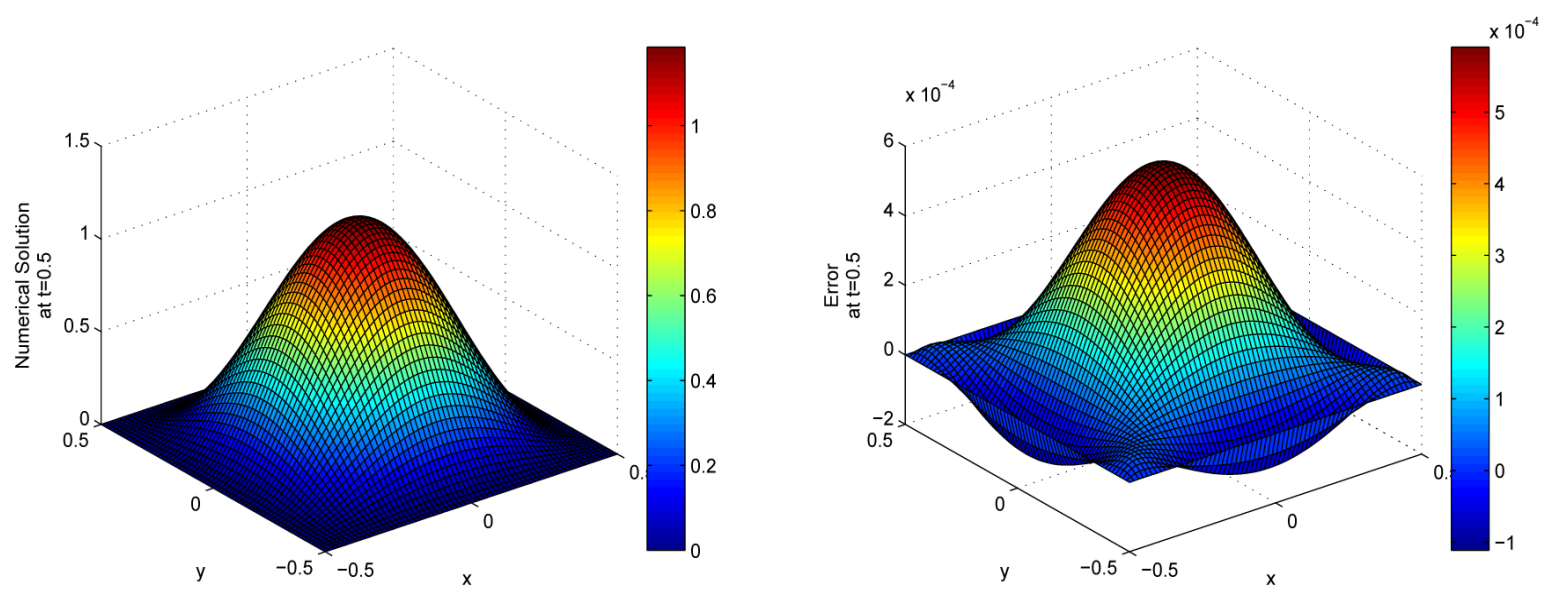

Figure 3. Numerical solution (left) and error (right) for $\alpha=1: 9$ at $t=0.5$. 


$$
\left\{\begin{array}{l}
\frac{\partial}{\partial t} u=a\left(\frac{\partial^{\alpha} u}{\partial|x|^{\alpha}}+\frac{\partial^{\alpha} u}{\partial|y|^{\alpha}}\right)+f, \text { in } Q_{T}=(0, T] \times \Omega \\
\left.u(x, y, t)\right|_{\partial \Omega}=0 \\
u(x, y, 0)=0, \text { on } \Omega=(-0.5,0.5) \times(-0.5,0.5)
\end{array}\right.
$$

Let $f=10 \cos (\pi x) \cos (\pi y)\left[\cos (t)+2 a \pi^{2} \sin (t)\right]$ and we know if $\alpha=2$ the equation reduces to classical diffusion equation which has exact solution $u=10 \sin (t) \cos (\pi x) \cos (\pi y)$. Now, we take $a=1, T=0.5$, $\tau=0.01, h=1 / 32$ and $\alpha=1.1,1.4,1.7,1.99$ respectively to show the character of the system in Figure 4 and Figure 5. From the numerical experiments we conclude that the bigger $\alpha$ is, the smaller $u(x, y, T)$ is. I.e., the process of diffusion becomes faster on the whole.

Example 3. In order to compare the difference between fractional diffusion and classical diffusion, consider the following equation with homogeneous boundary condition:

$$
\left\{\begin{array}{l}
\frac{\partial}{\partial t} u=\frac{1}{10}\left(\frac{\partial^{\alpha} u}{\partial|x|^{\alpha}}+\frac{\partial^{\alpha} u}{\partial|y|^{\alpha}}\right), \text { in } Q_{T}=(0,1] \times \Omega \\
\left.u(x, y, t)\right|_{\partial \Omega}=0, \Omega=(-0.5,0.5) \times(-0.5,0.5)
\end{array}\right.
$$
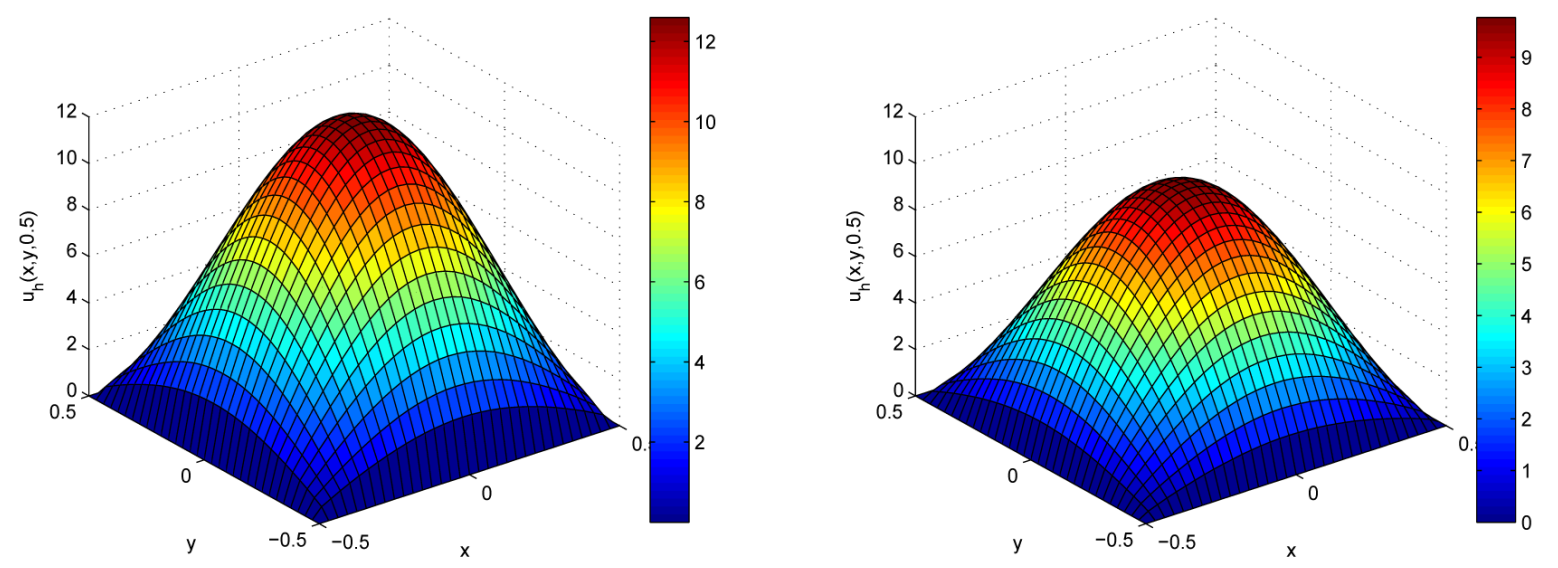

Figure 4. Numerical solution for $\alpha=1: 1$ (left) and $\alpha=1: 4$ (right).
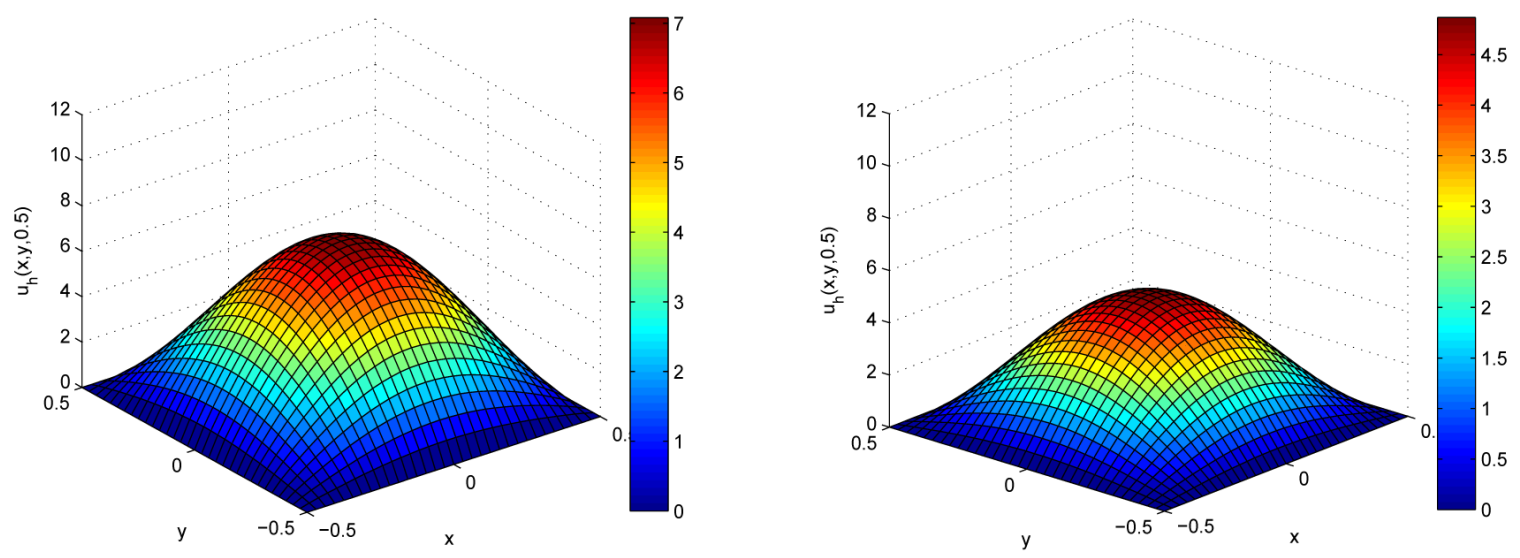

Figure 5. Numerical solution for $\alpha=1: 7$ (left) and $\alpha=1: 99$ (right). 
where $u$ represents concentration and the diffusion coefficient is $\frac{1}{10}$. The initial value of $u$ satisfies

$$
u(x, y, 0)= \begin{cases}250, & \text { if } 0.5|x|+|y| \leq 0.1 \\ 0, & \text { otherwise }\end{cases}
$$

which means the initial concentration concentrates in a rhombus. We take $\alpha=1.4$ and $\alpha=2$ in the above equation respectively, then plot isolines at $t=0.01, t=0.05, t=0.15, t=0.35, t=0.65$ and $t=1$ in the following images of Figure 6 ( $\alpha=1.4$ on the left side and $\alpha=2$ on the right side).
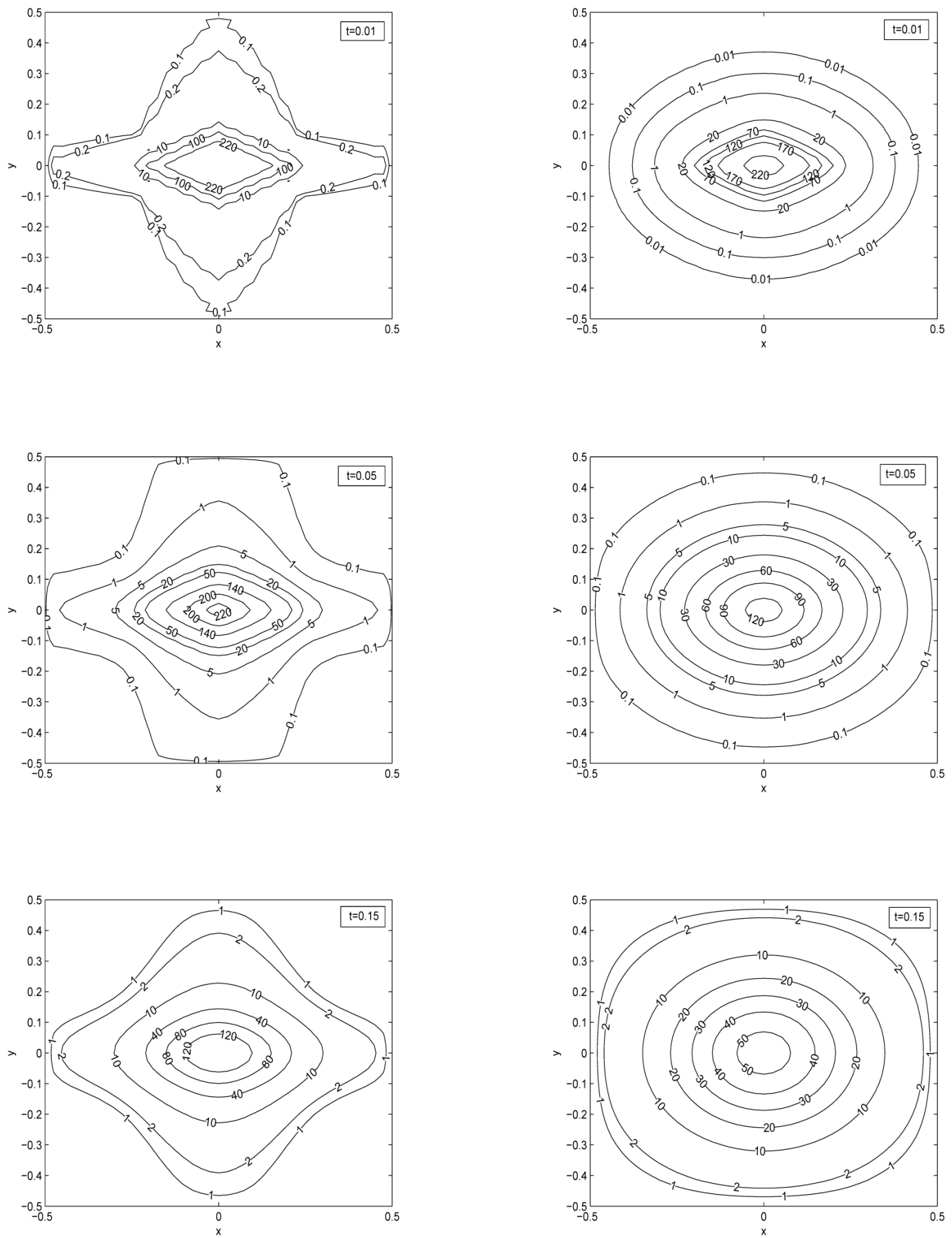

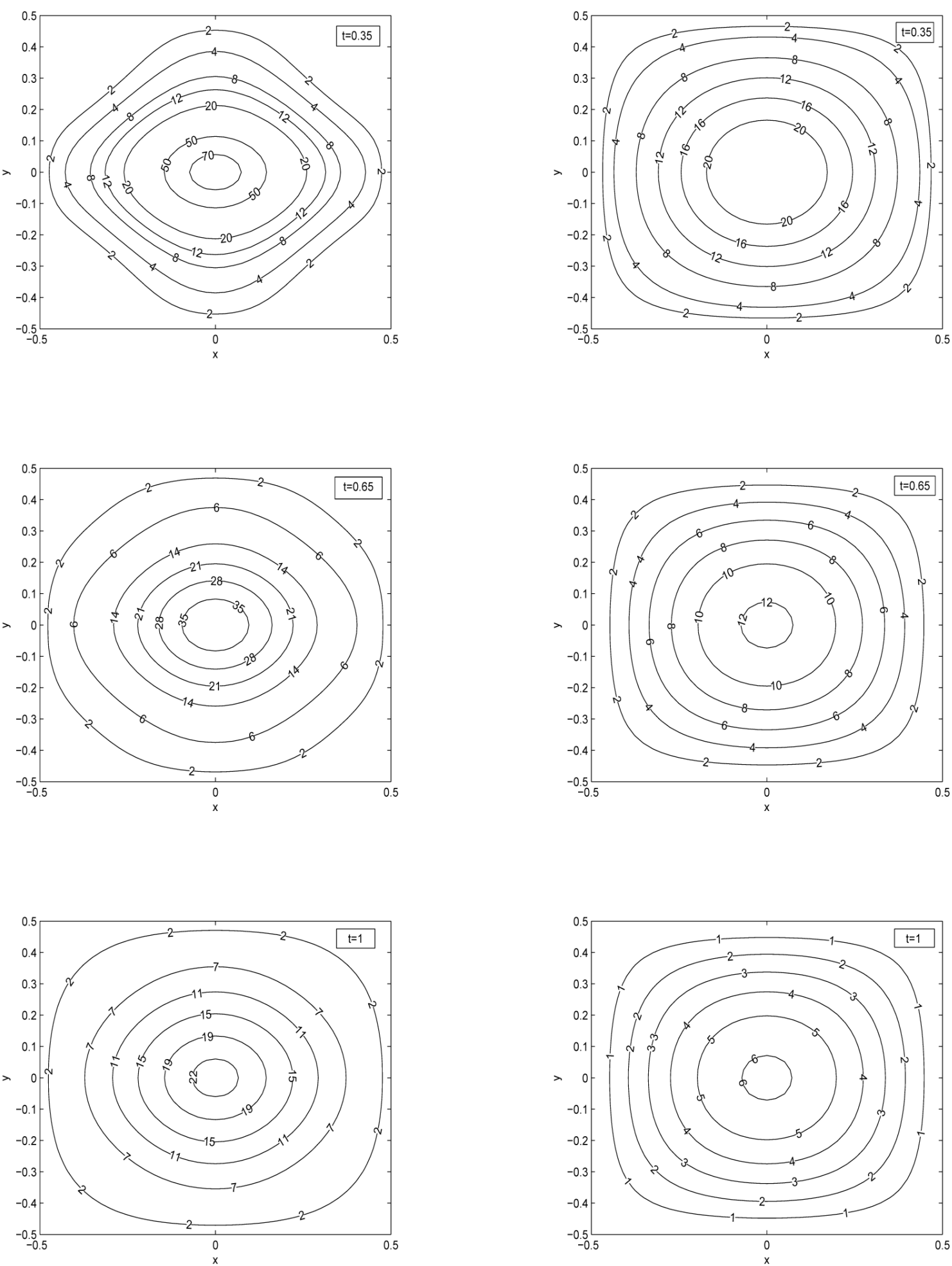

Figure 6. Contour maps of $\alpha=1: 4$ (left) and $\alpha=2$ (right) at specified time.

We note that the initial condition in the fractional system affect wider area than integer order in a short period of time by comparing the first two contour maps. Moreover, the diffusion under the influence of initial condition last longer in the fractional system. So at $t=0.35$, the diffusion in classical system is almost uniform in every 
direction but this state needs more time to reach in the fractional system (see the left map at $t=1$ ).

\section{Conclusion}

Many different numerical methods for fractional convection-diffusion equation have been discussed by researchers in recent 10 years. In this paper, we discussed one kind of space-fractional diffusion equation which could be derived through replacing the second order derivative of $x$ and $y$ by corresponding Riesz fractional derivative in the classical diffusion equation. A numerical approximation for the equation was presented by using C-N technique in time direction and Galerkin finite method in space. Furthermore, a detailed stability and convergence analysis was carried out for the fully discrete system. Then, some numerical examples were given and the differences between fractional and classical diffusion were presented. It is known that the stiffness matrix of fractional differential equation is rather complex, so to make the approach applicatory. We give the implementation of computational aspect. However, because of the non-local property of fractional derivative, the stiffness matrix is not sparse (almost dense) which challenges the computational resources.

\section{Acknowledgements}

The authors were supported by the National Natural Science Foundation of China under Project 51174236, and the National Basic Research Program of China under Project 2011CB606306.

\section{References}

[1] Metzler, R. and Klafter, J. (2004) The Restaurant at the End of the Random Walk: Recent Developments in the Description of Anomalous Transport by Fractional Dynamics. Journal of Physics A: Mathematical and General, 37, R161R208. http://dx.doi.org/10.1088/0305-4470/37/31/R01

[2] Zaslavsky, G.M., Stevens, D. and Weitzner, H. (1993) Self-Similar Transport in Incomplete Chaos. Physical Review E, 48, 1683-1694. http://dx.doi.org/10.1103/PhysRevE.48.1683

[3] Metzler, R. and Klafter, J. (2000) The Random Walk’s Guide to Anomalous Diffusion: A Fractional Dynamics Approach. Physics Reports, 339, 1-77. http://dx.doi.org/10.1016/S0370-1573(00)00070-3

[4] Zaslavsky, G.M. (2002) Chaos, Fractional Kinetics, and Anomalous Transport. Physics Reports, 371, 461-580. http://dx.doi.org/10.1016/S0370-1573(02)00331-9

[5] Schumer, R., Benson, D.A., Meerschaert, M.M. and Baeumer, B. (2003) Multiscaling Fractional Advection-Dispersion Equations and Their Solutions. Water Resources Research, 39, 1022-1032. http://dx.doi.org/10.1029/2001WR001229

[6] Schumer, R., Benson, D.A., Meerschaert, M.M. and Wheatcraft, S.W. (2001) Eulerian Derivation of the Fractional Advection-Dispersion Equation. Journal of Contaminant Hydrology, 48, 69-88.

http://dx.doi.org/10.1016/S0169-7722(00)00170-4

[7] Tadjeran, C., Meerschaert, M.M. and Scheffler, H.P. (2006) A Second-Order Accurate Numerical Approximation for the Fractional Diffusion Equation. Journal of Computational Physics, 213, 205-213. http://dx.doi.org/10.1016/j.jcp.2005.08.008

[8] Tadjeran, C. and Meerschaert, M.M. (2007) A Second-Order Accurate Numerical Method for the Two-Dimensional Fractional Diffusion Equation. Journal of Computational Physics, 220, 813-823. http://dx.doi.org/10.1016/j.jcp.2006.05.030

[9] Sousa, E. (2011) Numerical Approximations for Fractional Diffusion Equations via Splines. Computers and Mathematics with Applications, 62, 938-944. http://dx.doi.org/10.1016/j.camwa.2011.04.015

[10] Li, X. and Xu, C. (2009) A Space-Time Spectral Method for the Time Fractional Diffusion Equation. SIAM Journal on Numerical Analysis, 47, 2108-2131.

[11] Xu, Q. and Hesthaven, J.S. (2013) Discontinuous Galerkin Method for Fractional Convection-Diffusion Equations. http://arxiv.org/abs/1304.6047

[12] Jin, B., Lazarov, R., Liu, Y. and Zhou, Z. (2015) The Galerkin Finite Element Method for a Multi-Term Time-Fractional Diffusion Equation. Journal of Computational Physics, 281, 825-843. http://dx.doi.org/10.1016/j.jcp.2014.10.051

[13] Chaves, A.S. (1998) A Fractional Diffusion Equation to Describe Lévy Flight. Physics Letters A, 239, 13-16. http://dx.doi.org/10.1016/S0375-9601(97)00947-X

[14] Benson, D.A., Wheatcraft, S.W. and Meerschaert, M.M. (2000) Application of a Fractional Advection-Dispersion Equation. Water Resources Research, 36, 1403-1412. http://dx.doi.org/10.1029/2000WR900031 
[15] Benson, D.A., Wheatcraft, S.W. and Meerschaert, M.M. (2000) The Fractional Order Governing Equation of Lévy Motion. Water Resources Research, 36, 1413-1423. http://dx.doi.org/10.1029/2000WR900032

[16] Zhang, H., Liu, F. and Anh, V. (2010) Galerkin Finite Element Approximation of Symmetric Space-Fractional Partial Differential Equations. Applied Mathematics and Computation, 217, 2534-2545. http://dx.doi.org/10.1016/j.amc.2010.07.066

[17] Sousa, E. (2012) A Second Order Explicit Finite Difference Method for the Fractional Advection Diffusion Equation. Computers and Mathematics with Applications, 64, 3141-3152. http://dx.doi.org/10.1016/j.camwa.2012.03.002

[18] Liu, F., Zhuang, P., Turner, I., Anh, V. and Burrage, K. (2015) A Semi-Alternating Direction Method for a 2-D Fractional FitzHugh-Nagumo Monodomain Model on an Approximate Irregular Domain. Journal of Computational Physics, 293, 252-263.

[19] Zeng, F., Liu, F., Li, C., Burrage, K., Turner, I. and Anh, V. (2014) Crank-Nicolson ADI Spectral Method for the TwoDimensional Riesz Space Fractional Nonlinear Reaction-Diffusion Equation. SIAM Journal on Numerical Analysis, 52, 2599-2622. http://dx.doi.org/10.1137/130934192

[20] Wang, H. and Du, N. (2013) A Fast Finite Difference Method for Three-Dimensional Time-Dependent Space-Fractional Diffusion Equations and Its Efficient Implementation. Journal of Computational Physics, 253, 50-63. http://dx.doi.org/10.1016/j.jcp.2013.06.040

[21] Wang, H. and Du, N. (2014) Fast Alternating-Direction Finite Difference Methods for Three-Dimensional SpaceFractional Diffusion Equations. Journal of Computational Physics, 258, 305-318. http://dx.doi.org/10.1016/j.jcp.2013.10.040

[22] Ervin, V.J. and Roop, J.P. (2006) Variational Formulation for the Stationary Fractional Advection Dispersion Equation. Numerical Methods for Partial Differential Equations, 22, 558-576. http://dx.doi.org/10.1002/num.20112

[23] Ervin, V.J. and Roop, J.P. (2007) Variational Solution of Fractional Advection Dispersion Equations on Bounded Domains in $R^{d}$. Numerical Methods for partial Differential Equations, 23, 256-281. http://dx.doi.org/10.1002/num.20169

[24] Podlubny, I. (1999) Fractional Differential Equations. Academic Press, Waltham.

[25] Muslih, S.I. and Agrawal, O.P. (2010) Riesz Fractional Derivatives and Fractional Dimensional Space. International Journal of Theoretical Physics, 49, 270-275. http://dx.doi.org/10.1007/s10773-009-0200-1

[26] El-Sayed, A.M.A. and Gaber, M. (2006) On the Finite Caputo and Finite Riesz Derivatives. Electronic Journal of Theoretical Physics, 3, 81-95.

[27] Brenner, S. and Scott, L.R. (1994) The Mathematical Theory of Finite Element Methods. Springer-Verlag, New York. http://dx.doi.org/10.1007/978-1-4757-4338-8

[28] Roop, J.P. (2006) Computational Aspects of FEM Approximation of Fractional Advection Dispersion Equations on Bounded Domains in $R^{2}$. Journal of Computational and Applied Mathematics, 193, 243-268. 


\section{Appendix}

Here, we give the computational details of case 5 to case 8 . It is analogous for case 1 to case 4 . To begin with, we introduce one formula which is used frequently in the procedure of computing the inner product and can be derived directly from the definition of beta function by integral transformation:

$$
\int_{\xi}^{\eta}(\eta-x)^{r}(x-\xi)^{s} \mathrm{~d} x=B(r+1, s+1)(\eta-\xi)^{r+s+1},
$$

where $B(\cdot, \cdot)$ is the beta function and $r, s>-1$.

In the following analysis, we always denote $K=\operatorname{supp}\left({ }_{a} D_{x}^{\alpha / 2} \psi_{i} \cdot{ }_{x} D_{b}^{\alpha / 2} \psi_{j}\right)$.

Case 5: $p=n-1, q=m$, i.e. $j=i-1$

It is obvious that $K=K_{1} \cup K_{4}$. With noticing that $\psi_{i}$ and $\psi_{j}$ are both symmetrical about the straight lines $y=y_{m}$, we have

$$
\begin{aligned}
\left({ }_{a} D_{x}^{\alpha / 2} \psi_{i},{ }_{x} D_{b}^{\alpha / 2} \psi_{j}\right)= & 2\left(\frac{1}{4 l_{1} \Gamma(2-\alpha / 2)}\right)^{2} \int_{y_{m-1}}^{y_{m}}\left(1+\frac{y-y_{0}^{1}}{l_{2}}\right)^{2} \mathrm{~d} y \\
& \times \int_{x_{n-1}}^{x_{n}}\left(x-x_{n-1}\right)^{1-\alpha / 2}\left(x_{n}-x\right)^{1-\alpha / 2} \mathrm{~d} x \\
& =2\left(\frac{1}{4 l_{1} \Gamma(2-\alpha / 2)}\right)^{2} \frac{8}{3} l_{2} \cdot B(2-\alpha / 2,2-\alpha / 2)\left(2 l_{1}\right)^{3-\alpha / 2} \\
& =\frac{l_{2} l_{1}^{1-\alpha}}{3 \Gamma(4-\alpha)} 2^{3-\alpha}
\end{aligned}
$$

Case 6: $p=n, q=m$, i.e. $\quad j=i$

In this cas $K=K_{1} \cup K_{2} \cup K_{3} \cup K_{4}$. Consider the inner product in $K_{1}$.

$$
\begin{aligned}
& \int_{K_{1}} D_{x}^{\alpha / 2} \psi_{i x} D_{b}^{\alpha / 2} \psi_{j} \mathrm{~d} x \\
& =\left(\frac{1}{4 l_{1} \Gamma(2-\alpha / 2)}\right)^{2} \int_{y_{m-1}}^{y_{m}}\left(1+\frac{y-y_{0}^{1}}{l_{2}}\right) \mathrm{d} y \cdot \int_{x_{n-1}}^{x_{n}}\left(x-x_{n-1}\right)^{1-\alpha / 2}\left[\left(x_{n+1}-x\right)^{1-\alpha / 2}-2\left(x_{n}-x\right)^{1-\alpha / 2}\right] \mathrm{d} x \\
& =\left(\frac{1}{4 l_{1} \Gamma(2-\alpha / 2)}\right)^{2} \frac{8}{3} l_{2}\left\{\int_{x_{n-1}}^{x_{n}}\left[\left(x_{n+1}-x\right)\left(x-x_{n-1}\right)\right]^{1-\alpha / 2} \mathrm{~d} x-2 \int_{x_{n-1}}^{x_{n}}\left[\left(x_{n}-x\right)\left(x-x_{n-1}\right)\right]^{1-\alpha / 2} \mathrm{~d} x\right\} \\
& =\left(\frac{1}{4 l_{1} \Gamma(2-\alpha / 2)}\right)^{2} \frac{8}{3} l_{2}\left[\frac{1}{2} B(2-\alpha / 2,2-\alpha / 2)\left(4 l_{1}\right)^{3-\alpha}-2 B(2-\alpha / 2,2-\alpha / 2)\left(2 l_{1}\right)^{3-\alpha}\right] \\
& =\frac{l_{2} l_{1}^{1-\alpha}}{12 \Gamma(4-\alpha)}\left(4^{3-\alpha}-4 \cdot 2^{3-\alpha}\right)
\end{aligned}
$$

Because the two basis functions are symmetrical about the straight lines $x=x_{n}$ and $y=y_{m}$, so we can derive that

$$
\begin{aligned}
\int_{K_{a}} D_{x}^{\alpha / 2} \psi_{i{ }_{x}} D_{b}^{\alpha / 2} \psi_{j} \mathrm{~d} x & =4 \int_{K_{1 a}} D_{x}^{\alpha / 2} \psi_{i x} D_{b}^{\alpha / 2} \psi_{j} \mathrm{~d} x \\
& =\frac{l_{2} l_{1}^{1-\alpha}}{3 \Gamma(4-\alpha)}\left(4^{3-\alpha}-4 \cdot 2^{3-\alpha}\right)
\end{aligned}
$$

Case 7: $p=n+1, q=m$, i.e. $\quad j=i+1$

It is easy to see $K=\left[x_{n-1}, x_{n+2}\right] \times\left[y_{m-1}, y_{m+1}\right]$, with noticing that the $\psi_{i}, \psi_{j}$ are both symmetrical about the straight lines $y=y_{m}$, then we can get 


$$
\begin{aligned}
& \int_{K a} D_{x}^{\alpha / 2} \psi_{i x} D_{b}^{\alpha / 2} \psi_{j} \mathrm{~d} x=2 \cdot\left(\frac{1}{4 l_{1} \Gamma(2-\alpha / 2)}\right)^{2} \cdot \int_{y_{m-1}}^{y_{m}}\left(1+\frac{y-y_{0}^{1}}{l_{2}}\right)^{2} \mathrm{~d} y \\
& \quad \cdot\left\{\int_{x_{n-1}}^{x_{n}}\left[\left(x_{n+2}-x\right)^{1-\alpha / 2}-2\left(x_{n+1}-x\right)^{1-\alpha / 2}+\left(x_{n}-x\right)^{1-\alpha / 2}\right]\left(x-x_{n-1}\right)^{1-\alpha / 2} \mathrm{~d} x\right. \\
& \quad+\int_{x_{n}}^{x_{n+1}}\left[\left(x_{n+2}-x\right)^{1-\alpha / 2}-2\left(x_{n+1}-x\right)^{1-\alpha / 2}\right]\left[\left(x-x_{n-1}\right)^{1-\alpha / 2}-2\left(x-x_{n}\right)^{1-\alpha / 2}\right] \mathrm{d} x \\
& \left.\quad+\int_{x_{n}}^{x_{n+1}}\left(x_{n+2}-x\right)^{1-\alpha / 2}\left[\left(x-x_{n-1}\right)^{1-\alpha / 2}-2\left(x-x_{n}\right)^{1-\alpha / 2}+\left(x-x_{n+1}\right)^{1-\alpha / 2}\right] \mathrm{d} x\right\} \\
& =\frac{16}{3} l_{2}\left(\frac{1}{4 l_{1} \Gamma(2-\alpha / 2)}\right)^{2} B(2-\alpha / 2,2-\alpha / 2)\left[\left(6 l_{1}\right)^{3-\alpha}-4 \cdot\left(4 l_{1}\right)^{3-\alpha}+6 \cdot\left(2 l_{1}\right)^{3-\alpha}\right] \\
& =\frac{l_{2} l_{1}^{1-\alpha}}{3 \Gamma(4-\alpha)}\left(6^{3-\alpha}-4 \cdot 4^{3-\alpha}+6 \cdot 2^{3-\alpha}\right)
\end{aligned}
$$

Case 8: $p=n+1+k, q=m$, i.e. $j=i+1+k$

First, we consider the case of $k=0$. In this case $K=\left[x_{n-1}, x_{n+3}\right] \times\left[y_{m-1}, y_{m+1}\right]$.

$$
\begin{aligned}
& \int_{K a} D_{x}^{\alpha / 2} \psi_{i x} D_{b}^{\alpha / 2} \psi_{j} \mathrm{~d} x=2 \cdot\left(\frac{1}{4 l_{1} \Gamma(2-\alpha / 2)}\right)^{2} \cdot \int_{y_{m-1}}^{y_{m}}\left(1+\frac{y-y_{0}^{1}}{l_{2}}\right)^{2} \mathrm{~d} y \\
& \quad \cdot\left\{\int_{x_{n-1}}^{x_{n}}\left[\left(x_{n+3}-x\right)^{1-\alpha / 2}-2\left(x_{n+2}-x\right)^{1-\alpha / 2}+\left(x_{n+1}-x\right)^{1-\alpha / 2}\right]\left(x-x_{n-1}\right)^{1-\alpha / 2} \mathrm{~d} x\right. \\
& \quad+\int_{x_{n}}^{x_{n+1}}\left[\left(x_{n+3}-x\right)^{1-\alpha / 2}-2\left(x_{n+2}-x\right)^{1-\alpha / 2}+\left(x_{n+1}-x\right)^{1-\alpha / 2}\right]\left[\left(x-x_{n-1}\right)^{1-\alpha / 2}-2\left(x-x_{n}\right)^{1-\alpha / 2}\right] \mathrm{d} x \\
& \quad+\int_{x_{n+1}}^{x_{n+2}}\left[\left(x_{n+3}-x\right)^{1-\alpha / 2}-2\left(x_{n+2}-x\right)^{1-\alpha / 2}\right]\left[\left(x-x_{n-1}\right)^{1-\alpha / 2}-2\left(x-x_{n}\right)^{1-\alpha / 2}+\left(x-x_{n+1}\right)^{1-\alpha / 2}\right] \mathrm{d} x \\
& \left.\quad+\int_{x_{n+2}}^{x_{n+3}}\left(x_{n+3}-x\right)^{1-\alpha / 2}\left[\left(x-x_{n-1}\right)^{1-\alpha / 2}-2\left(x-x_{n}\right)^{1-\alpha / 2}+\left(x-x_{n+1}\right)^{1-\alpha / 2}\right] \mathrm{d} x\right\} \\
& =\frac{16}{3} l_{2}\left(\frac{1}{4 l_{1} \Gamma(2-\alpha / 2)}\right)^{2} B(2-\alpha / 2,2-\alpha / 2)\left[\left(8 l_{1}\right)^{3-\alpha}-4 \cdot\left(6 l_{1}\right)^{3-\alpha}+6 \cdot\left(4 l_{1}\right)^{3-\alpha}-4 \cdot\left(2 l_{1}\right)^{3-\alpha}\right] \\
& =\frac{l_{2} l_{1}^{1-\alpha}}{3 \Gamma(4-\alpha)}\left(8^{3-\alpha}-4 \cdot 6^{3-\alpha}+6 \cdot 4^{3-\alpha}-4 \cdot 2^{3-\alpha}\right)
\end{aligned}
$$

By induction, we can conclude that for $k=1,2, \cdots$,

$$
\left({ }_{a} D_{x}^{\alpha / 2} \psi_{i x} D_{b}^{\alpha / 2} \psi_{j}\right)=\frac{l_{2} l_{1}^{1-\alpha}}{3 \Gamma(4-\alpha)}\left[(8+2 k)^{3-\alpha}-4 \cdot(6+2 k)^{3-\alpha}+6 \cdot(4+2 k)^{3-\alpha}-4 \cdot(2+2 k)^{3-\alpha}+(2 k)^{3-\alpha}\right] .
$$

